\title{
GRÖBNER BASES AND GRADINGS FOR PARTIAL DIFFERENCE IDEALS
}

\author{
ROBERTO LA SCALA
}

\begin{abstract}
In this paper we introduce a working generalization of the theory of Gröbner bases for algebras of partial difference polynomials with constant coefficients. One obtains symbolic (formal) computation for systems of linear or non-linear partial difference equations arising, for instance, as discrete models or by the discretization of systems of differential equations. From an algebraic viewpoint, the algebras of partial difference polynomials are free objects in the category of commutative algebras endowed with the action by endomorphisms of a monoid isomorphic to $\mathbb{N}^{r}$. Then, the investigation of Gröbner bases in this context contributes also to the current research trend consisting in studying polynomial rings under the action of suitable symmetries that are compatible with effective methods. Since the algebras of difference polynomials are not Noetherian, we propose in this paper a theory for grading them that provides a Noetherian subalgebra filtration. This implies that the variants of Buchberger's algorithm we developed for difference ideals terminate in the finitely generated graded case when truncated up to some degree. Moreover, even in the non-graded case, we provide criterions for certifying completeness of eventually finite Gröbner bases when they are computed within sufficiently large bounded degrees. We generalize also the concepts of homogenization and saturation, and related algorithms, to the context of difference ideals. The feasibility of the proposed methods is shown by an implementation in Maple that is the first to provide computations for systems of non-linear partial difference equations. We make use of a test set based on the discretization of concrete systems of non-linear partial differential equations.
\end{abstract}

\section{INTRODUCTION}

An important idea at the intersection of many algebraic theories consists in studying algebraic structures under the action of operators of different natures, typically automorphisms and derivations. Classical roots of this idea can be found clearly in invariant and representation theory, as well as in the study of polynomial identities satisfied by associative algebras. Recently, topics like algebraic statistic [4, 16] or entanglement theory [24] have given new impulse and applications to the research on such themes. Another fundamental source of inspiration is the theory of differential and difference algebras introduced in the pioneeristic work of Ritt [25, 26] and afterwards developed by Kolchin [17, Cohn [6], Levin [23] and many others. From the point of view of computational methods, starting from the algorithms proposed by Ritt himself, a considerable advancement can be recorded in

Received by the editor December 14, 2011 and, in revised form, November 6, 2012, May 30, 2013 and July 9, 2013.

2010 Mathematics Subject Classification. Primary 12H10; Secondary 13P10, 16W22, 16W50.

Key words and phrases. Partial difference equations, Gröbner bases, actions on algebras, gradings on algebras.

The author was partially supported by Università di Bari. 
the differential case (see for instance [27]). Much less has been achieved for the algebras of difference polynomials where working algorithms can be found mainly in the linear case [12. Nevertheless, the interest for such computations is relevant because of applications in the discretization of systems of differential equations like the automatic generation of finite difference schemes or the consistency analysis of finite difference approximations $9,11,21$. The present paper contributes to this research trend by concerning the development of effective methods for systems of linear or non-linear partial difference equations with constant coefficients. We provide also an implementation of such methods which is the first to allow computations in the non-linear case. Specifically, we generalize the theory of Gröbner bases and related algorithms for ideals of the algebra of partial difference polynomials. We are able to do this in a general and systematic way, by defining classes of suitable monomial orderings, by extending Buchberger's algorithm and the concept of grading to difference ideals, by defining truncated homogeneous computations and even by introducing a suitable notion of homogenization for such ideals. The first contributions to such a theory can be found in 9, 19, 20. In particular, owing to the notion of "letterplace correspondence/embedding" introduced in [18 20], note that Gröbner bases computations for ideals of the free associative algebra are a subclass of the same computations for ideals of the algebra of ordinary difference polynomials.

The algebras of partial difference polynomials are free algebras in the class of commutative algebras that are invariant under the action by endomorphisms of a monoid isomorphic to $\mathbb{N}^{r}$. Then, the study of Gröbner bases for such algebras belongs to the general investigation of computational methods for commutative rings or modules that have suitable symmetries. Moreover, from the viewpoint of applications, the algebras of partial difference polynomials are fundamental structures in the formal theory of partial difference equations where a set of unknown multivariate functions is assumed algebraically independent together with all partial shifts of them. To provide symbolic computation for systems of such equations is hence essential to introduce Gröbner bases methods. Based on a suitable definition of monomial orderings that are compatible with shift action, and the description of large classes of them, the present paper introduces variants of Buchberger's algorithm for partial difference ideals. These procedures take advantage of the monoid symmetry essentially by killing all S-polynomials in an orbit except for a minimal one. Note that the algebras of difference polynomials are not Noetherian since they are polynomial rings in an infinite number of variables and hence termination is not generally guaranteed for the proposed algorithms. With the aim of improving this situation, we define suitable gradings that are compatible with the monoid action and provide filtrations of the algebra of partial difference polynomials with finitely generated subalgebras. We obtain therefore the termination for finitely generated graded difference ideals when computations are performed within some bounded degree. For non-graded ideals, except for monomial orderings compatible with such gradings, we also prove criterions able to certify that a Gröbner basis computation performed over a suitable finite set of variables that is within a sufficiently large degree, is a complete one. Finally, the paper generalizes the notion of homogenization and saturation to difference ideals with respect to the given gradings and provides the algorithms to perfom these ideal operations. As a byproduct, one obtains an alternative algorithm to compute Gröbner bases of non-graded difference ideals via 
homogeneous computations. By means of an implementation in Maple, all of these methods are finally tested on difference ideals obtained by the discretization of systems of non-linear differential equations.

\section{Algebras of partial difference polynomials}

Fix $K$ as any field and let $\Sigma$ be a monoid (semigroup with identity) that we denote multiplicatively. Let $A$ be a commutative $K$-algebra and denote $\operatorname{End}_{K}(A)$ the monoid of $K$-algebra endomorphisms of $A$. We call $A$ a $\Sigma$-invariant algebra or briefly a $\Sigma$-algebra if there is a monoid homomorphism $\rho: \Sigma \rightarrow \operatorname{End}_{K}(A)$. In this case, we denote $\sigma \cdot x=\rho(\sigma)(x)$, for all $\sigma \in \Sigma$ and $x \in A$. Let $A, B$ be $\Sigma$-algebras and $\varphi: A \rightarrow B$ be a $K$-algebra homomorphism. We say that $\varphi$ is a $\Sigma$-algebra homomorphism if $\varphi(\sigma \cdot x)=\sigma \cdot \varphi(x)$, for all $\sigma \in \Sigma$ and $x \in A$. Let $A$ be a $\Sigma$-algebra and let $I \subset A$ be an ideal. We call $I$ a $\Sigma$-invariant ideal or simply a $\Sigma$-ideal if $\Sigma \cdot I \subset I$. Clearly, all kernels of $\Sigma$-algebra homomorphisms are $\Sigma$-ideals.

Definition 2.1. Let $A$ be a $\Sigma$-algebra and let $X \subset A$ be a subset. We say that $A$ is $\Sigma$-generated by $X$ if $A$ is generated by $\Sigma \cdot X$ as $K$-algebra. In other words, $A$ coincides with the smallest $\Sigma$-subalgebra of $A$ containing $X$. In the same way, one defines $\Sigma$-generation for the $\Sigma$-ideals.

In the category of $\Sigma$-invariant algebras one can define free objects. In fact, let $X$ be a set and denote $x(\sigma)$ each element $(x, \sigma)$ of the product set $X(\Sigma)=X \times \Sigma$. Define $P=K[X(\Sigma)]$ the polynomial algebra in the commuting variables $x(\sigma)$. For any element $\sigma \in \Sigma$ consider the $K$-algebra endomorphism $\bar{\sigma}: P \rightarrow P$ such that $x(\tau) \mapsto x(\sigma \tau)$, for all $x(\tau) \in X(\Sigma)$. Then, one has a faithful monoid representation $\rho: \Sigma \rightarrow \operatorname{End}_{K}(P)$ such that $\rho(\sigma)=\bar{\sigma}$ and hence $P$ is a $\Sigma$-algebra. Note that if $\Sigma$ is a left-cancellative monoid then all maps $\rho(\sigma)$ are injective.

Proposition 2.2. Let $A$ be a $\Sigma$-algebra and let $f: X \rightarrow A$ be any map. Then there is a unique $\Sigma$-algebra homomorphism $\varphi: P \rightarrow A$ such that $\varphi(x(1))=f(x)$, for all $x \in X$.

Proof. It is sufficient to define $\varphi(x(\sigma))=\sigma \cdot f(x)$, for all $x \in X$ and $\sigma \in \Sigma$. In fact, one has $\varphi(\tau \cdot x(\sigma))=\varphi(x(\tau \sigma))=\tau \sigma \cdot f(x)=\tau \cdot(\sigma \cdot f(x))=\tau \cdot \varphi(x(\sigma))$, for any $\tau \in \Sigma$.

Definition 2.3. We call $P=K[X(\Sigma)]$ the free $\Sigma$-algebra generated by $X$. In fact, $P$ is $\Sigma$-generated by the subset $X(1)=\left\{x_{i}(1) \mid x_{i} \in X\right\}$.

In other words, the algebra $P$ is an essential tool in the theory of $\Sigma$-algebras because any such algebra $A$ that is $\Sigma$-generated by a set $X$ can be obtained as a quotient $\Sigma$-algebra $P / I$, where $I$ is a $\Sigma$-ideal of $P$. For instance, from the viewpoint of computational methods, if one develops them for $P$, then such methods can be extended to any quotient $P / I$ as is done in the classical theory of Gröbner bases for affine algebras. Note also that if $\Sigma$ is defined as the monoid $\operatorname{Inc}(\mathbb{N})=\{f: \mathbb{N} \rightarrow$ $\mathbb{N} \mid f$ strictly increasing $\}$, or some power of this, one obtains an environment for computations in algebraic statistic [4,16.

Now we want to go in the direction of developing fundamental structures for symbolic (formal) computation on systems of partial difference equations with constant coefficients. From now on, we assume that $X=\left\{x_{0}, x_{1}, \ldots\right\}$ is a finite or countable set and $\Sigma$ is a free commutative monoid generated by a finite set, say $\left\{\sigma_{1}, \ldots, \sigma_{r}\right\}$. 
Then, we consider the free $\Sigma$-algebra $P=K[X(\Sigma)]$. Note that $(\Sigma, \cdot)$ is a cancellative monoid isomorphic to $\left(\mathbb{N}^{r},+\right)$ and the monomorphisms $\rho(\sigma): P \rightarrow P$ have infinite order for all $\sigma \neq 1$. For any $x_{i}(\sigma) \in X(\Sigma)$, we call $i$ and $\sigma$, respectively, the index and the weight of the variable $x_{i}(\sigma)$. If we put $X(\sigma)=\left\{x_{i}(\sigma) \mid x_{i} \in X\right\}$ and $x_{i}(\Sigma)=\left\{x_{i}(\sigma) \mid \sigma \in \Sigma\right\}$ one clearly has $P=\bigotimes_{\sigma \in \Sigma} K[X(\sigma)]=\bigotimes_{x_{i} \in X} K\left[x_{i}(\Sigma)\right]$, where all subalgebras $K[X(\sigma)]$ are isomorphic to $K[X]$ and all subalgebras $K\left[x_{i}(\Sigma)\right]$ to $K[\Sigma]$.

Definition 2.4. The free $\Sigma$-algebra $P=K[X(\Sigma)]\left(\Sigma=\left\langle\sigma_{1}, \ldots, \sigma_{r}\right\rangle\right)$ is called the algebra of partial difference polynomials with constant coefficients.

The motivation for such a name is in the formal theory of partial difference equations [6, 23. In this theory, in fact, the indeterminates $x_{i}(1)$ are by definition algebraically independent unknown functions $u_{i}\left(t_{1}, \ldots, t_{r}\right)$ in the variables $t_{j}$ and the maps $\rho\left(\sigma_{k}\right)$ are the shift operators $u_{i}\left(t_{1}, \ldots, t_{r}\right) \mapsto u_{i}\left(t_{1}, \ldots, t_{k}+h, \ldots, t_{r}\right)$ where $h$ is a parameter (mesh step). If $\sigma=\prod_{i} \sigma_{i}^{\alpha_{i}}$ then the indeterminates $x_{i}(\sigma)=$ $\sigma \cdot x_{i}(1)$ correspond to the (algebraically independent) shifted functions $u_{i}\left(t_{1}+\right.$ $\left.\alpha_{1} h, \ldots, t_{r}+\alpha_{r} h\right)=\sigma \cdot u_{i}\left(t_{1}, \ldots, t_{r}\right)$. Then, a $\Sigma$-ideal $I \subset P$ is also called a partial difference ideal and a $\Sigma$-basis of $I$ corresponds to a system of partial difference equations in the unknown functions $u_{i}\left(t_{1}, \ldots, t_{r}\right)$. One uses the term ordinary difference when $r=1$. Note that the algebras of difference polynomials are not Noetherian rings since they are polynomial rings in an infinite number of variables. One therefore has that difference ideals have bases or $\Sigma$-bases which are generally infinite.

In the next sections we generalize the Gröbner basis theory to the free $\Sigma$-algebra $P=K[X(\Sigma)]$ of partial difference polynomials. Clearly, one reobtains the classical theory when $\Sigma=\{1\}(r=0)$ that is $P=K[X]$. The starting point is to define monomial orderings of $P$ which are compatible with the action of the monoid $\Sigma$.

\section{MonOMial $\Sigma$-ORDERINGS}

Denote by $M=\operatorname{Mon}(P)$ the set of all monomials of $P$. Note that even if the set $X(\Sigma)$ is infinite (in fact countable), one can endow $P$ by monomial orderings. This is an important consequence of Higman's Lemma [15] which can be stated in the following way (see for instance [1, Corollary 2.3 and remarks at the beginning of page 5175).

Proposition 3.1. Let $\prec$ be a total ordering on $M$ such that

(i) $1 \preceq m$ for all $m \in M$;

(ii) $\prec$ is compatible with multiplication on $M$; that is, if $m \prec n$, then $t m \prec t n$, for any $m, n, t \in M$.

Then $\prec$ is also a well-ordering of $M$ that is a monomial ordering of $P$ if and only if the restriction of $\prec$ to the variables set $X(\Sigma)$ is a well-ordering.

Clearly, it is easy to construct well-orderings for the set $X(\Sigma)$ which is in bijective correspondence to $\mathbb{N}^{r+1}$. Note that the monoid $\Sigma$ stabilizes the monomials set $M$ since it stabilizes $X(\Sigma)$. Thus, we introduce the following notion.

Definition 3.2. Let $\prec$ be a monomial ordering of $P$. We call $\prec$ a (monomial) $\Sigma$-ordering of $P$ if $\prec$ is compatible with the $\Sigma$-action on $M$, that is, $m \prec n$ implies that $\sigma \cdot m \prec \sigma \cdot n$ for all $m, n \in M$ and $\sigma \in \Sigma$. 
A straightforward consequence of this definition is the following result.

Proposition 3.3. Let $\prec$ be a monomial $\Sigma$-ordering of $P$. Then $m \preceq \sigma \cdot m$ for all $m \in M$ and $\sigma \in \Sigma$.

Proof. By contradiction, assume that there are $m, \sigma$ such that $m \succ \sigma \cdot m$. Then $\sigma \cdot m \succ \sigma^{2} \cdot m$ and by induction one obtains the infinite descending chain $m \succ$ $\sigma \cdot m \succ \sigma^{2} \cdot m \succ \ldots$ which contradicts that $\prec$ is a well-ordering.

The orderings on the variables set $X(\Sigma)$ that can be extended to monomial $\Sigma$-orderings are as follows.

Definition 3.4. Let $\prec$ be a well-ordering of $X(\Sigma)$. We call $\prec$ a (variable) $\Sigma$ ranking of $P$ if $\prec$ is compatible with the $\Sigma$-action on $X(\Sigma)$, that is, $u \prec v$ implies that $\sigma \cdot u \prec \sigma \cdot v$ for all $u, v \in X(\Sigma)$ and $\sigma \in \Sigma$.

As for Proposition 3.3, we have that if $\prec$ is a $\Sigma$-ranking, then $u \preceq \sigma \cdot u$ for all $u \in X(\Sigma)$ and $\sigma \in \Sigma$. Moreover, if $X$ is a finite set, then condition $u \preceq \sigma \cdot u$ for all $u, \sigma$ together with $\Sigma$-compatibility implies that $\prec$ is a well-ordering by applying Dickson's Lemma (or Higman's Lemma) to $\Sigma$ which is isomorphic to $\mathbb{N}^{r}$. However, note that in this paper the set $X$ may be also countable.

Owing to the decompositions $X(\Sigma)=\bigcup_{\sigma \in \Sigma} X(\sigma)=\bigcup_{x_{i} \in X} x_{i}(\Sigma)$ of the variables set of the ring $P$, we can define $\Sigma$-rankings of $P$ in a natural way. Denote by $Q$ the monoid $K$-algebra defined by the free commutative monoid $\Sigma=\left\langle\sigma_{1}, \ldots, \sigma_{r}\right\rangle$. In other words, $Q=K\left[\sigma_{1}, \ldots, \sigma_{r}\right]$ is the polynomial algebra in the commutative variables $\sigma_{i}$. From now on, we assume that $\Sigma$ is endowed with a monomial ordering $<$ of $Q$. By abuse, we call < a monomial ordering of $\Sigma$.

Definition 3.5. Fix $<$ a monomial ordering of $\Sigma$. For all $x_{i}(\sigma), x_{j}(\tau) \in X(\Sigma)$, we define:

(i) $x_{i}(\sigma) \prec x_{j}(\tau)$ if and only if $\sigma<\tau$ or $\sigma=\tau$ and $i<j$. In other words, $X(\sigma) \prec X(\tau)$ when $\sigma<\tau$.

(ii) $x_{i}(\sigma) \prec^{\prime} x_{j}(\tau)$ if and only if $i<j$ or $i=j$ and $\sigma<\tau$. In other words, $x_{i}(\Sigma) \prec^{\prime} x_{j}(\Sigma)$ when $i<j$.

Clearly, $\prec$ and $\prec^{\prime}$ are both $\Sigma$-rankings of $P$ that we call, respectively, weight and index $\Sigma$-ranking defined by a monomial ordering of $\Sigma$.

For all $x_{i} \in X$ and $\sigma \in \Sigma$ denote $P(\sigma)=K[X(\sigma)], M(\sigma)=\operatorname{Mon}(P(\sigma))$ and $P\left(x_{i}\right)=K\left[x_{i}(\Sigma)\right], M\left(x_{i}\right)=\operatorname{Mon}\left(P\left(x_{i}\right)\right)$. Owing to the tensor decompositions $P=\bigotimes_{\sigma \in \Sigma} P(\sigma)=\bigotimes_{x_{i} \in X} P\left(x_{i}\right)$, one has that a monomial $m \in M$ can be uniquely written as $m=m\left(\delta_{1}\right) \cdots m\left(\delta_{k}\right)=m\left(x_{i_{1}}\right) \cdots m\left(x_{i_{l}}\right)$, where $m\left(\delta_{p}\right) \in$ $M\left(\delta_{p}\right), m\left(x_{i_{p}}\right) \in M\left(x_{i_{p}}\right)$ and $\delta_{1}>\ldots>\delta_{k}, i_{1}>\ldots>i_{l}$. By means of such presentations we can define block monomial orderings of $P$ extending weight and index ranking. Recall that $\rho: \Sigma \rightarrow \operatorname{End}_{K}(P)$ is the faithful monoid representation defined by the action of $\Sigma$ over $P$. For any $\sigma \in \Sigma$ one has that the map $\rho(\sigma)$ defines an isomorphism between the monoids $M(1), M(\sigma)$ and hence between the algebras $P(1), P(\sigma)$. In other words, we have $M(\sigma)=\sigma \cdot M(1), P(\sigma)=\sigma \cdot P(1)$.

Definition 3.6. Fix $\prec$ a monomial ordering of the subalgebra $P(1) \subset P$ and extend it to all subalgebras $P(\sigma)(\sigma \in \Sigma)$ by the isomorphisms $\rho(\sigma)$. In other words, we put $\sigma \cdot m \prec \sigma \cdot n$ if and only if $m \prec n$, for any $m, n \in M(1)$. Then, for all $m, n \in M, m=m\left(\delta_{1}\right) \cdots m\left(\delta_{k}\right), n=n\left(\delta_{1}\right) \cdots n\left(\delta_{k}\right)$ with $\delta_{1}>\ldots>\delta_{k}$ we define 
$m \prec{ }_{w} n$ if and only if $m\left(\delta_{j}\right)=n\left(\delta_{j}\right)$ if $j<i$ and $m\left(\delta_{i}\right) \prec n\left(\delta_{i}\right)$ for some $1 \leq i \leq k$. Clearly, the restriction of $\prec_{w}$ to the variables of $P$ is just the weight $\Sigma$-ranking.

Proposition 3.7. The ordering $\prec_{w}$ is a $\Sigma$-ordering of $P$.

Proof. Note that if $m=m\left(\delta_{1}\right) \cdots m\left(\delta_{k}\right) \in M$ with $m\left(\sigma_{i}\right) \in M\left(\sigma_{i}\right)$ and $\delta_{1}>$ $\ldots>\delta_{k}$ then $\sigma \cdot m=m\left(\sigma \delta_{1}\right) \cdots m\left(\sigma \delta_{k}\right)$, where $m\left(\sigma \delta_{i}\right)=\sigma \cdot m\left(\delta_{i}\right) \in M\left(\sigma \delta_{i}\right)$ and $\sigma \delta_{1}>\ldots>\sigma \delta_{k}$ since $<$ is a monomial ordering of $\Sigma$. Assume $m \prec_{w} n$ that is $m\left(\delta_{j}\right)=n\left(\delta_{j}\right)$ for $j<i$ and $m\left(\delta_{i}\right) \prec n\left(\delta_{i}\right)$. Clearly $m\left(\sigma \delta_{j}\right)=n\left(\sigma \delta_{j}\right)$ for $j<i$ and one has $m\left(\delta_{i}\right) \prec n\left(\delta_{i}\right)$ if and only if $m(1) \prec n(1)$ if and only if $m\left(\sigma \delta_{i}\right) \prec n\left(\sigma \delta_{i}\right)$. Then, we conclude that $\sigma \cdot m \prec_{w} \sigma \cdot n$.

Note that we have also a monoid faithful representation $\phi: \mathbb{N} \rightarrow \operatorname{End}_{K}(P)$ such that the endomorphism $\phi(i)$ is defined as $x_{j}(\sigma) \mapsto x_{i+j}(\sigma)$ for any $i, j \geq 0$ and $\sigma \in \Sigma$. Clearly $\phi(i)$ induces isomorphism between the monoids $M\left(x_{0}\right), M\left(x_{i}\right)$ and the algebras $P\left(x_{0}\right), P\left(x_{i}\right)$. The algebra $P\left(x_{0}\right)$ can be easily endowed with a $\Sigma$ ordering. For instance, since $P\left(x_{0}\right)=\bigotimes_{\sigma \in \Sigma} K\left[x_{0}(\sigma)\right]$ one can define a lexicographic ordering as in Definition 3.6

Definition 3.8. Fix $\prec$ a monomial $\Sigma$-ordering of the subalgebra $P\left(x_{0}\right) \subset P$ and extend it to all subalgebras $P\left(x_{i}\right)\left(x_{i} \in X\right)$ by the isomorphisms $\phi(i)$. For any $m, n \in M, m=m\left(x_{i_{1}}\right) \cdots m\left(x_{i_{k}}\right), n=n\left(x_{i_{1}}\right) \cdots n\left(x_{i_{k}}\right)$ with $i_{1}>\ldots>i_{k}$ we put $m \prec_{i} n$ if and only if $m\left(x_{i_{q}}\right)=n\left(x_{i_{q}}\right)$ if $q<p$ and $m\left(x_{i_{p}}\right) \prec n\left(x_{i_{p}}\right)$ for some $1 \leq p \leq k$. Note that the restriction of $\prec_{i}$ to the variables of $P$ is the index $\sum$-ranking.

Proposition 3.9. The ordering $\prec_{i}$ is a $\Sigma$-ordering of $P$.

Proof. Note that if $m=m\left(x_{i_{1}}\right) \cdots m\left(x_{i_{k}}\right) \in M$ with $m\left(x_{i_{p}}\right) \in M\left(x_{i_{p}}\right)$ and $i_{1}>$ $\ldots>i_{k}$ then $\sigma \cdot m=m^{\prime}\left(x_{i_{1}}\right) \cdots m^{\prime}\left(x_{i_{k}}\right)$ where $m^{\prime}\left(x_{i_{p}}\right)=\sigma \cdot m\left(x_{i_{p}}\right) \in M\left(x_{i_{p}}\right)$. Suppose $m \prec_{i} n$, that is, $m\left(x_{i_{q}}\right)=n\left(x_{i_{q}}\right)$ if $q<p$ and $m\left(x_{i_{p}}\right) \prec n\left(x_{i_{p}}\right)$. We have clearly that $m^{\prime}\left(x_{i_{q}}\right)=n^{\prime}\left(x_{i_{q}}\right)$. Moreover, since $\prec$ is a $\Sigma$-ordering of $P\left(x_{0}\right)$ and therefore of $P\left(x_{i_{p}}\right)$, one has also $m^{\prime}\left(x_{i_{p}}\right) \prec n^{\prime}\left(x_{i_{p}}\right)$ that is $\sigma \cdot m \prec{ }_{i} \sigma \cdot n$.

We call the above monomial $\Sigma$-orderings $\prec_{w}, \prec_{i}$ of $P$, respectively, weight $\Sigma$ ordering defined by a monomial ordering of $P(1)$ and index $\Sigma$-ordering of $P$ defined by a monomial $\Sigma$-ordering of $P\left(x_{0}\right)$. Clearly, both of these orderings depend also on a monomial ordering of $\Sigma$. Note that index $\Sigma$-orderings are suitable for generation of finite difference schemes for partial differential equations [10,11]. The weight $\Sigma$ orderings are instead compatible with the gradings of the $\Sigma$-algebra $P$, introduced in Section 6. For this reason they are suitable for obtaining complete Gröbner bases from truncated computations (see Proposition 6.14).

To make things more explicit, we now give an example of a weight and an index $\Sigma$-ordering. Fix $X=\{x, y, z\}$ and $\Sigma=\left\langle\sigma_{1}, \sigma_{2}\right\rangle$. To simplify the notation, we identify the monoid $(\Sigma, \cdot)$ with $\left(\mathbb{N}^{2},+\right)$ by means of the isomorphism $\sigma_{1}^{i} \sigma_{2}^{j} \mapsto(i, j)$. Then, we fix the degrevlex monomial ordering on $\Sigma$ with $\sigma_{1}>\sigma_{2}$, that is,

$$
\ldots>(2,0)>(1,1)>(0,2)>(1,0)>(0,1)>(0,0)
$$

and assume $P(x)=K[x(i, j) \mid i, j \geq 0]$ is endowed with the lex monomial ordering such that

$$
\ldots \succ x(2,0) \succ x(1,1) \succ x(0,2) \succ x(1,0) \succ x(0,1) \succ x(0,0) .
$$


Finally, we also fix the lex ordering on $P(0,0)=K[x(0,0), y(0,0), z(0,0)]$ with $x(0,0) \succ y(0,0) \succ z(0,0)$. By isomorphisms, one has clearly the same ordering on $P(y), P(z)$ and $P(i, j)=K[x(i, j), y(i, j), z(i, j)]$, for all $i, j \geq 0,(i, j) \neq(0,0)$. Then, a weight $\Sigma$-ordering is defined on $P=K[x(i, j), y(i, j), z(i, j) \mid i, j \geq 0]$ as the block monomial ordering corresponding to the tensor decomposition

$$
P=\ldots \otimes P(2,0) \otimes P(1,1) \otimes P(0,2) \otimes P(1,0) \otimes P(0,1) \otimes P(0,0) .
$$

In a similar way, one defines an index $\Sigma$-ordering on $P$ owing to the decomposition

$$
P=P(x) \otimes P(y) \otimes P(z) .
$$

Similar $\Sigma$-orderings have been used for the examples contained in Section 5 and 8 and for the computational experiments presented in Section 9 (see also the Appendix).

\section{GRÖBNER $\Sigma$-BASES}

From now on, we consider $P$ endowed with a monomial $\Sigma$-ordering $\prec$. Let $f=\sum_{i} c_{i} m_{i} \in P$ with $m_{i} \in M, c_{i} \in K, c_{i} \neq 0$. We denote as usual $\operatorname{lm}(f)=m_{k}=$ $\max _{\prec}\left\{m_{i}\right\}, \operatorname{lc}(f)=c_{k}$ and $\operatorname{lt}(f)=\operatorname{lc}(f) \operatorname{lm}(f)$. If $G \subset P$ we write

$$
\operatorname{lm}(G)=\{\operatorname{lm}(f) \mid f \in G, f \neq 0\}
$$

and we denote as $\operatorname{LM}(G)$ the ideal of $P$ generated by $\operatorname{lm}(G)$.

Proposition 4.1. Let $G \subset P$. Then $\operatorname{lm}(\Sigma \cdot G)=\Sigma \cdot \operatorname{lm}(G)$. In particular, if $I$ is a $\Sigma$-ideal of $P$, then $\operatorname{LM}(I)$ is also a $\Sigma$-ideal.

Proof. Since $P$ is endowed with a $\Sigma$-ordering, one has that $\operatorname{lm}(\sigma \cdot f)=\sigma \cdot \operatorname{lm}(f)$ for any $f \in P, f \neq 0$ and $\sigma \in \Sigma$. Then $\Sigma \cdot \operatorname{lm}(I)=\operatorname{lm}(\Sigma \cdot I) \subset \operatorname{lm}(I)$ and therefore $\operatorname{LM}(I)=\langle\operatorname{lm}(I)\rangle$ is a $\Sigma$-ideal.

Definition 4.2. Let $I \subset P$ be a $\Sigma$-ideal and $G \subset I$. We call $G$ a Gröbner $\Sigma$-basis of $I$ if $\operatorname{lm}(G)$ is a $\Sigma$-basis of $\operatorname{LM}(I)$. In other words, $\Sigma \cdot G$ is a Gröbner basis of $I$ as $P$-ideal.

Since the monoid $\Sigma$ is assumed isomorphic to $\mathbb{N}^{r}$, that is, $\Sigma$-ideals are partial difference ideals, we may say that Gröbner $\Sigma$-bases are partial difference Gröbner bases [9]. Another possible name is $\Sigma$-equivariant Gröbner bases [4]. Simplicity and generality lead us to the previous definition that has already appeared in [20].

Let $f, g \in P, f, g \neq 0$ and put $\operatorname{lt}(f)=c m, \operatorname{lt}(g)=d n$ with $m, n \in M$ and $c, d \in K$. If $l=\operatorname{lcm}(m, n)$ we define as usual the $S$-polynomial spoly $(f, g)=(l / \mathrm{cm}) f-(l / d n) g$. Clearly $\operatorname{spoly}(f, g)=-\operatorname{spoly}(g, f)$ and $\operatorname{spoly}(f, f)=0$.

Proposition 4.3. For all $f, g \in P, f, g \neq 0$ and for any $\sigma \in \Sigma$ one has $\sigma$. $\operatorname{spoly}(f, g)=\operatorname{spoly}(\sigma \cdot f, \sigma \cdot g)$.

Proof. Since $\Sigma$ acts on the variables set $X(\Sigma)$ by injective maps, it is sufficient to note that $\sigma \cdot \operatorname{lcm}(m, n)=\operatorname{lcm}(\sigma \cdot m, \sigma \cdot n)$ for all $m, n \in M$ and $\sigma \in \Sigma$.

The following definition is a standard tool in Gröbner bases theory.

Definition 4.4. Let $f \in P, f \neq 0$ and $G \subset P$. If $f=\sum_{i} f_{i} g_{i}$ with $f_{i} \in P, g_{i} \in G$ and $\operatorname{lm}(f) \succeq \operatorname{lm}\left(f_{i}\right) \operatorname{lm}\left(g_{i}\right)$ for all $i$, we say that $f$ has a Gröbner representation with respect to $G$. 
Note that, if $f=\sum_{i} f_{i} g_{i}$ is a Gröbner representation, then $\sigma \cdot f=\sum_{i}\left(\sigma \cdot f_{i}\right)\left(\sigma \cdot g_{i}\right)$ is also a Gröbner representation, for any $\sigma \in \Sigma$. In fact, since $\prec$ is a $\Sigma$-ordering of $P$ one has that $\operatorname{lm}(f) \succeq \operatorname{lm}\left(f_{i}\right) \operatorname{lm}\left(g_{i}\right)$ implies that $\operatorname{lm}(\sigma \cdot f)=\sigma \cdot \operatorname{lm}(f) \succeq$ $\left(\sigma \cdot \operatorname{lm}\left(f_{i}\right)\right)\left(\sigma \cdot \operatorname{lm}\left(g_{i}\right)\right)=\operatorname{lm}\left(\sigma \cdot f_{i}\right) \operatorname{lm}\left(\sigma \cdot g_{i}\right)$ for all $i$. A celebrated result from Bruno Buchberger [5] is the following.

Proposition 4.5 (Buchberger's criterion). Let $G$ be a basis of the ideal $I \subset P$. Then, $G$ is a Gröbner basis of $I$ if and only if for all $f, g \in G, f, g \neq 0$ the $S$ polynomial spoly $(f, g)$ has a Gröbner representation with respect to $G$.

Usually the above result (see for instance [8]) is stated when $P$ is a polynomial algebra with a finite number of variables and $G$ is a finite set. In fact, such assumptions are not needed since Noetherianity is not used in the proof, but only the existence of a monomial ordering for $P$. See also the comprehensive Bergman's paper [2] where the "Diamond Lemma" is proved without any restriction on the finiteness of the variables set. We now want to prove a generalization of Buchberger's criterion for Gröbner $\Sigma$-bases of $P$. For this purpose it is useful to introduce the following notation.

Definition 4.6. Let $\sigma=\prod_{i} \sigma_{i}^{\alpha_{i}}, \tau=\prod_{i} \sigma_{i}^{\beta_{i}} \in \Sigma$. We denote $\operatorname{gcd}(\sigma, \tau)=\prod_{i} \sigma_{i}^{\gamma_{i}}$ where $\gamma_{i}=\min \left(\alpha_{i}, \beta_{i}\right)$, for any $i$.

Proposition 4.7 ( $\Sigma$-criterion). Let $G$ be a $\Sigma$-basis of a $\Sigma$-ideal $I \subset P$. Then $G$ is a Gröbner $\Sigma$-basis of $I$ if and only if for all $f, g \in G, f, g \neq 0$ and for any $\sigma, \tau \in \Sigma$ such that $\operatorname{gcd}(\sigma, \tau)=1$, the $S$-polynomial $\operatorname{spoly}(\sigma \cdot f, \tau \cdot g)$ has a Gröbner representation with respect to $\Sigma \cdot G$.

Proof. We prove that $\Sigma \cdot G$ is a Gröbner basis of $I$ and we make use of Proposition 4.5. Then, consider any pair of elements $\sigma \cdot f, \tau \cdot g \in \Sigma \cdot G$ where $f, g \in$ $G, f, g \neq 0$ and $\sigma, \tau \in \Sigma$. Write $\delta=\operatorname{gcd}(\sigma, \tau)$ and hence $\sigma=\delta \sigma^{\prime}, \tau=\delta \tau^{\prime}$ with $\sigma^{\prime}, \tau^{\prime} \in \Sigma, \operatorname{gcd}\left(\sigma^{\prime}, \tau^{\prime}\right)=1$. By Proposition 4.3 we have $\operatorname{spoly}(\sigma \cdot f, \tau \cdot g)=$ $\delta \cdot \operatorname{spoly}\left(\sigma^{\prime} \cdot f, \tau^{\prime} \cdot g\right)$. By hypothesis, assume that spoly $\left(\sigma^{\prime} \cdot f, \tau^{\prime} \cdot g\right)=h=\sum_{\nu} f_{\nu}\left(\nu \cdot g_{\nu}\right)$, with $\nu \in \Sigma, f_{\nu} \in P, g_{\nu} \in G$, is a Gröbner representation with respect to $\Sigma \cdot G$. Since $\prec$ is a $\Sigma$-ordering of $P$, we conclude that we have also the Gröbner representation $\operatorname{spoly}(\sigma \cdot f, \tau \cdot g)=\delta \cdot h=\sum_{\nu}\left(\delta \cdot f_{\nu}\right)\left(\delta \nu \cdot g_{\nu}\right)$.

For the purpose of obtaining an effective Buchberger algorithm from the above criterion, note that all usual criteria (product criterion, chain criterion, etc.) can be used also in such a procedure. In particular, the arguments contained in the proof of Proposition 6.14 (see comments after this proof) imply that for any pair of elements $f, g \in G$ and for all $\sigma, \tau \in \Sigma$ there are only a finite number of Spolynomials $\operatorname{spoly}(\sigma \cdot f, \tau \cdot g)$ satisfying both the criteria $\operatorname{gcd}(\sigma, \tau)=1$ and $\operatorname{gcd}(\sigma$. $\operatorname{lm}(f), \tau \cdot \operatorname{lm}(g)) \neq 1$.

A standard subroutine in Buchberger's algorithm follows; see Algorithm 4.1.

Note that the termination of REDUCE is provided since $\prec$ is a monomial ordering of $P$. In particular, even if $G$ is an infinite set, there are only a finite number of elements $g \in G, g \neq 0$ such that $\operatorname{lm}(g)$ divides $\operatorname{lm}(h)$ and hence $\operatorname{lm}(g) \preceq \operatorname{lm}(h)$. It is well known that if $\operatorname{ReducE}(f, G)=0$ then $f$ has a Gröbner representation with respect to $G$. Moreover, if $\operatorname{Reduce}(f, G)=h \neq 0$, then clearly one has $\operatorname{ReducE}(f, G \cup\{h\})=0$. Therefore, from Proposition 4.7 and product criterion we get immediately the correctness of the following algorithm. 

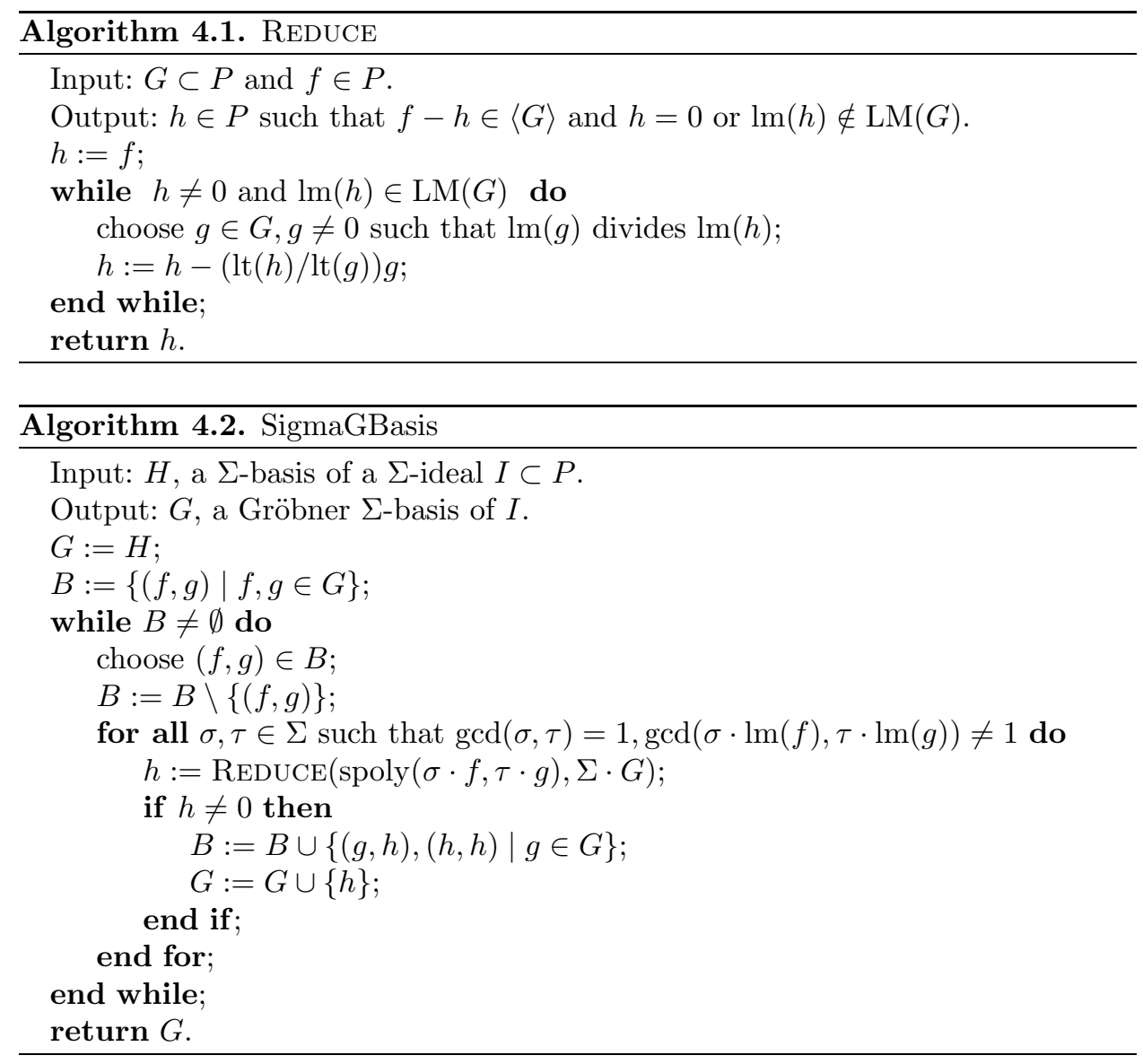

Note that Algorithm 4.2 can be viewed as a variant of the usual Buchberger's procedure applied for the basis $\Sigma \cdot H$, where an additional criterion to avoid "useless pairs" is given by Proposition 4.7. Unfortunately, owing to Non-Noetherianity of the ring $P$, the termination of SigmaGBAsis is not provided in general and this is, in fact, one of the main problems in differential/difference algebra. Precisely, even if a $\Sigma$-ideal $I \subset P$ has a finite $\Sigma$-basis this may not be the case for the initial $\Sigma$-ideal $\operatorname{LM}(I)$, that is, all Gröbner $\Sigma$-bases of $I$ are infinite sets. Despite this bad general case, in Section 6 we introduce suitable gradings for the algebra $P$ which provides that truncated versions of the algorithm SigmaGBASIS with homogeneous input stops in a finite number of steps. Finally, note that some variant of SigmaGBASIS appeared in [9] and before in [19,20, for the ordinary difference case. In fact, the notion of "letterplace correspondence/embedding" introduced in these latter papers strictly relates non-commutative Gröbner bases to Gröbner $\Sigma$-bases of ordinary difference ideals (see also [18]).

\section{An illustrative example}

In this section we apply the algorithm SigmaGBAsis to a simple example in order to provide a concrete computation with it. Let $X=\{x, y\}, \Sigma=\left\langle\sigma_{1}, \sigma_{2}\right\rangle$ and consider the algebra of partial difference polynomials $P=K[X(\Sigma)]$. To simplify the 
notation, we identify the monoid $(\Sigma, \cdot)$ with $\left(\mathbb{N}^{2},+\right)$ by means of the isomorphism $\sigma_{1}^{i} \sigma_{2}^{j} \mapsto(i, j)$. Then, we denote the variables of $P$ as $x(i, j), y(i, j)$, for all $i, j \geq 0$. We consider now the $\Sigma$-ideal (difference ideal) $I \subset P$ that is $\Sigma$-generated by the difference polynomials

$$
\begin{aligned}
& g_{1}=y(1,1) y(1,0)-2 x(0,1)^{2}, \\
& g_{2}=y(2,0)+x(0,0) x(1,0) .
\end{aligned}
$$

In other words, this $\Sigma$-basis (difference basis) encodes a system of non-linear difference equations with constant coefficients in two unknown bivariate functions. By symbolic (formal) computations, we want to substitute this system with a completion of it, namely a Gröbner $\Sigma$-basis. We may want to do this for the purposes of checking membership of other equations to the $\Sigma$-ideal, elimination of unknowns, etc. The main problem is that such a basis may be infinite, but it is not the case for this example. We then fix the degrevlex ordering on $\Sigma$ with $\sigma_{1}>\sigma_{2}$ that is on $\mathbb{N}^{2}$ where $(1,0)>(0,1)$. Moreover, we consider the lex monomial ordering on $K[x(0,0), y(0,0)]$ with $x(0,0) \succ y(0,0)$. A weight $\Sigma$-ordering (Definition 3.6) is hence defined on $P=\bigotimes_{(i, j) \in \mathbb{N}^{2}} K[x(i, j), y(i, j)]$ as a block monomial ordering. In practice, it is the lexicographic monomial ordering based on the following weight $\sum$-ranking

$$
\begin{aligned}
& \ldots \succ x(2,0) \succ y(2,0) \succ x(1,1) \succ y(1,1) \succ x(0,2) \succ y(0,2) \succ x(1,0) \\
& \succ y(1,0) \succ x(0,1) \succ y(0,1) \succ x(0,0) \succ y(0,0) .
\end{aligned}
$$

We use this monomial $\Sigma$-ordering of $P$ for computing a Gröbner $\Sigma$-basis of $I$. Such a basis consists of the elements $g_{1}, g_{2}$ together with the difference polynomials

$$
\begin{aligned}
& g_{3}=y(1,2) x(0,1)^{2}-y(1,0) x(0,2)^{2}, \\
& g_{4}=2 x(1,1)^{2}-x(0,0) x(1,0) x(0,1) x(1,1) .
\end{aligned}
$$

Note that in all these elements the first monomial is the leading one with respect to the given ordering of $P$. Let us see how the algorithm SigmaGBAsis is able to obtain such Gröbner $\Sigma$-basis. Since the $\Sigma$-ideal $I$ is $\Sigma$-generated by $G=\left\{g_{1}, g_{2}\right\}$, then $I$ is generated as an ideal of $P$ by $\Sigma \cdot G$ that are the polynomials

$$
\begin{aligned}
& (i, j) \cdot g_{1}=y(i+1, j+1) y(i+1, j)-2 x(i, j+1)^{2}, \\
& (i, j) \cdot g_{2}=y(i+2, j)+x(i, j) x(i+1, j),
\end{aligned}
$$

for all $i, j \geq 0$. By applying the product criterion, we have to consider three kinds of S-polynomials

$$
\begin{aligned}
& \operatorname{spoly}\left((i, j+1) \cdot g_{1},(i, j) \cdot g_{1}\right)=(i, j) \cdot \operatorname{spoly}\left((0,1) \cdot g_{1}, g_{1}\right), \\
& \operatorname{spoly}\left((i+1, j) \cdot g_{1},(i, j) \cdot g_{2}\right)=(i, j) \cdot \operatorname{spoly}\left((1,0) \cdot g_{1}, g_{2}\right), \\
& \operatorname{spoly}\left((i+1, j+1) \cdot g_{1},(i, j+2) \cdot g_{2}\right)=(i, j+1) \cdot \operatorname{spoly}\left((1,0) \cdot g_{1},(0,1) \cdot g_{2}\right) .
\end{aligned}
$$

The $\Sigma$-criterion implies therefore that one has to reduce, with respect to the basis $\Sigma \cdot G$, just the S-polynomials

$$
\begin{aligned}
& s_{1}=\operatorname{spoly}\left((0,1) \cdot g_{1}, g_{1}\right), \\
& s_{2}=\operatorname{spoly}\left((1,0) \cdot g_{1}, g_{2}\right), \\
& s_{3}=\operatorname{spoly}\left((1,0) \cdot g_{1},(0,1) \cdot g_{2}\right) .
\end{aligned}
$$

The reduction of the S-polynomial $s_{1}$ leads to the new element $g_{3}$ and the current $\Sigma$-basis of $I$ is now $G=\left\{g_{1}, g_{2}, g_{3}\right\}$. The additional S-polynomials that survive to 
product and $\Sigma$-criterion are

$$
\begin{aligned}
& s_{4}=\operatorname{spoly}\left((0,1) \cdot g_{1}, g_{3}\right), \\
& s_{5}=\operatorname{spoly}\left((0,2) \cdot g_{1}, g_{3}\right), \\
& s_{6}=\operatorname{spoly}\left((0,2) \cdot g_{2},(1,0) \cdot g_{3}\right) .
\end{aligned}
$$

We have that $s_{4} \rightarrow 0$ and $s_{2} \rightarrow g_{4}$ with respect to $\Sigma \cdot G$. The $\Sigma$-basis is then $G=\left\{g_{1}, g_{2}, g_{3}, g_{4}\right\}$ and one has a new S-polynomial

$$
s_{7}=\operatorname{spoly}\left((1,0) \cdot g_{3}, g_{4}\right) \text {. }
$$

Finally, we have that all S-polynomials $s_{3}, s_{5}, s_{6}, s_{7}$ reduce to zero with respect to $\Sigma \cdot G$ and hence $G$ is a Gröbner $\Sigma$-basis of $I$. Note that $G$ is in fact a minimal such basis and also that in this simple example there is no use of the chain criterion that can always be applied together with the other criteria.

\section{Gradings of $P$ Compatible with $\Sigma$-aCtion}

We want now to introduce some gradings of the algebra $P=K[X(\Sigma)]$ which are compatible with $\Sigma$-action and formation of least common multiples in $M=$ $\operatorname{Mon}(P)$. As before, we fix a monomial order $<$ of $\Sigma$. We start by extending the structure $(\Sigma, \max , \cdot)$ in the following way.

Definition 6.1. Let 0 be an element disjoint with $\Sigma$ and put $\hat{\Sigma}=\Sigma \cup\{0\}$. Then we define a commutative idempotent monoid $(\hat{\Sigma},+)$ with identity 0 that extends the monoid $(\Sigma, \max )$ (with identity 1 ) by imposing that $0+\sigma=\sigma$, for any $\sigma \in \hat{\Sigma}$. Moreover, we define a commutative monoid $(\hat{\Sigma}, \cdot)$ with identity 1 extending the monoid $(\Sigma, \cdot)$ by putting $0 \cdot \sigma=0$, for all $\sigma \in \hat{\Sigma}$. Since multiplication clearly distributes over addition, one has that $(\hat{\Sigma},+, \cdot)$ is a commutative idempotent semiring, also known as commutative dioid [13].

Note that the faithful monoid representation $\rho: \Sigma \rightarrow \operatorname{End}_{K}(P)$ can be extended to $\hat{\Sigma}$ where $\rho(0): P \rightarrow P$ is the algebra endomorphism such that $x_{i}(\sigma) \mapsto 0$, for all $x_{i}(\sigma) \in X(\Sigma)$.

Definition 6.2. Let $\mathrm{w}: M \rightarrow \hat{\Sigma}$ be the unique mapping such that

(i) $\mathrm{w}(1)=0$;

(ii) $\mathrm{w}(m n)=\mathrm{w}(m)+\mathrm{w}(n)$, for any $m, n \in M$;

(iii) $\mathrm{w}\left(x_{i}(\sigma)\right)=\sigma$, for all $i \geq 0$ and $\sigma \in \Sigma$.

Note that (i),(ii) state that $\mathrm{w}$ is a monoid homomorphism from the free commutative monoid $(M, \cdot)$ to $(\hat{\Sigma},+)$. We call $\mathrm{w}$ the weight function of $P$.

More explicitly, if $m=x_{i_{1}}\left(\delta_{1}\right)^{\alpha_{1}} \cdots x_{i_{k}}\left(\delta_{k}\right)^{\alpha_{k}}$ is any monomial of $P$ different from 1 , then $\mathrm{w}(m)=\delta_{1}+\cdots+\delta_{k}=\max _{<}\left(\delta_{1}, \ldots, \delta_{k}\right)$. We denote $M_{\sigma}=\{m \in M \mid$ $\mathrm{w}(m)=\sigma\}$ and define $P_{\sigma} \subset P$ the subspace spanned by $M_{\sigma}$, for any $\sigma \in \hat{\Sigma}$. Because $\mathrm{w}:(M, \cdot) \rightarrow(\hat{\Sigma},+)$ is a monoid homomorphism one has that $P=\bigoplus_{\sigma \in \hat{\Sigma}} P_{\sigma}$ is a grading of the algebra $P$ over the commutative monoid $(\hat{\Sigma},+)$. If $f \in P_{\sigma}$ we say that $f$ is a w-homogeneous element and we put $\mathrm{w}(f)=\sigma$. Recall that for any $\sigma \in \Sigma$ we denoted $P(\sigma)=K[X(\sigma)]$ which is a subalgebra of $P=K[X(\Sigma)]$ isomorphic to $K[X]$. If we put $P(0)=P_{0}=K$ then one has that $P^{(\sigma)}=\bigoplus_{\tau \leq \sigma} P_{\sigma}=\bigotimes_{\tau \leq \sigma} P(\tau)$ is a subalgebra of $P$. In particular, we have that $P^{(1)}=P_{0} \oplus P_{1}=P(0) \otimes P(1)=$ $P(1)$ is isomorphic to the polynomial algebra $K[X]$. 
Definition 6.3. A monomial order $<$ of $\Sigma$ is said to be sequential if $\{\tau \in \Sigma \mid \tau \leq \sigma\}$ is a finite set, for all $\sigma \in \Sigma$.

It is important to note that if $X$ is a finite set and $<$ is a sequential ordering of $\Sigma$, then the sequence $\left\{P^{(\sigma)} \mid \sigma \in \hat{\Sigma}\right\}$ is a filtration of $P$ consisting of Noetherian subalgebras. For this reason, from now on we assume $\Sigma$ to be endowed with a sequential monomial ordering.

Proposition 6.4. The weight function satisfies the following properties:

(i) $\mathrm{w}(\sigma \cdot m)=\sigma \mathrm{w}(m)$, for any $\sigma \in \Sigma$ and $m \in M$;

(ii) $\mathrm{w}(\operatorname{lcm}(m, n))=\mathrm{w}(m n)=\mathrm{w}(m)+\mathrm{w}(n)$, for all $m, n \in M$. Then, $m \mid n$ implies that $\mathrm{w}(m) \leq \mathrm{w}(n)$.

Proof. If $m=1$ then $\mathrm{w}(\sigma \cdot m)=\mathrm{w}(m)=0=\sigma \mathrm{w}(m)$. If otherwise $m=$ $x_{i_{1}}\left(\delta_{1}\right)^{\alpha_{1}} \cdots x_{i_{k}}\left(\delta_{k}\right)^{\alpha_{k}}$ with $\delta_{1}>\ldots>\delta_{k}$ then $\sigma \cdot m=x_{i_{1}}\left(\sigma \delta_{1}\right)^{\alpha_{1}} \cdots x_{i_{k}}\left(\sigma \delta_{k}\right)^{\alpha_{k}}$ where $\sigma \delta_{1}>\ldots>\sigma \delta_{k}$ since $<$ is a monomial ordering of $\Sigma$. We conclude that $\mathrm{w}(\sigma \cdot m)=\sigma \delta_{1}=\sigma \mathrm{w}(m)$. To prove (ii) it is sufficient to note that the weight of a monomial does not depend on the exponents of the variables occurring in it.

Note that the property (i) implies that the map $\mathrm{w}$ is a homomorphism with respect to the action of $\Sigma$ on $M$ and $\hat{\Sigma}$. In other words, one has that $\sigma P_{\tau} \subset P_{\sigma \tau}$ for any $\sigma \in \Sigma, \tau \in \hat{\Sigma}$. Moreover, the property (ii) means that $\mathrm{w}$ is also a monoid homomorphism from $(M, \mathrm{lcm})$ to $(\hat{\Sigma},+)$.

Definition 6.5. Let $I$ be an ideal of $P$. We call $I$ a w-graded ideal if $I=\sum_{\sigma} I_{\sigma}$ with $I_{\sigma}=I \cap P_{\sigma}$. In this case, if $I$ is also a $\Sigma$-ideal, then $\sigma \cdot I_{\tau} \subset I_{\sigma \tau}$ for all $\sigma \in \Sigma, \tau \in \hat{\Sigma}$.

Owing to the w-grading of $P$, one can show that a truncated version of the algorithm SigmaGBAsis admits termination. If $f, g \in P, f \neq g$ are w-homogeneous elements then the S-polynomial $h=\operatorname{spoly}(f, g)$ is clearly w-homogeneous too. Moreover, by property (ii) of Proposition 6.4, we have that $\mathrm{w}(h)=\mathrm{w}(f)+\mathrm{w}(g)$ and hence if $\mathrm{w}(f), \mathrm{w}(g) \leq \delta$ then also $\mathrm{w}(h) \leq \delta$, for some $\delta \in \Sigma$. By means of this remark, one obtains immediately the following result.

Proposition 6.6 (Truncated termination over the weight). Let $I \subset P$ be $a$ wgraded $\Sigma$-ideal and fix $\delta \in \Sigma$. Assume $I$ has a w-homogeneous basis $H$ such that $H_{\delta}=\{f \in H \mid \mathrm{w}(f) \leq \delta\}$ is a finite set. Then there is a w-homogeneous Gröbner $\Sigma$-basis $G$ of $I$ such that $G_{\delta}$ is also a finite set. In other words, if we consider for the algorithm SigmaGBASIS a selection strategy of the S-polynomials based on their weights ordered by $<$, we obtain that the $\delta$-truncated version of SigmaGBASIS stops in a finite number of steps.

Proof. First, note that the algorithm SigmaGBAsis computes essentially a subset $G$ of a Gröbner basis $\Sigma \cdot G$ obtained by applying Buchberger's algorithm to the basis $\Sigma \cdot H$ of $I$. Moreover, by Proposition 6.4 the elements of $\Sigma \cdot H$ and hence of $\Sigma \cdot G$ are all w-homogeneous. Denote $H_{\delta}^{\prime}=\{\sigma \cdot f \mid \sigma \in \Sigma, f \in H, \sigma \mathrm{w}(f) \leq \delta\}$. Since $<$ is a sequential monomial order of $\Sigma$ and $H_{\delta}$ is a finite set one has that $H_{\delta}^{\prime}$ is also a finite set. We consider therefore $X_{\delta}$ the finite set of variables of $P$ occurring in the elements of $H_{\delta}^{\prime}$ and define $P_{(\delta)}=K\left[X_{\delta}\right] \subset P$. In fact, the $\delta$ truncated algorithm SigmaGBASIS computes a subset of a Gröbner basis of the ideal $I_{(\delta)} \subset P_{(\delta)}$ generated by $H_{\delta}^{\prime}$. By Noetherianity of the finitely generated polynomial ring $P_{(\delta)}$ we clearly obtain termination. 
Clearly the above result provides algorithmic solution to the ideal membership problem for finitely generated w-graded $\Sigma$-ideals. Note that if $r=0$, that is, $\Sigma=\{1\}$, then the algorithm SigmaGBAsis coincides with classical Buchberger's algorithm and Proposition 6.6 states that if $I$ is a finitely generated ideal of $P=$ $P_{0} \oplus P_{1}=K\left[x_{0}, x_{1}, \ldots\right]$ then $I$ has also a finite Gröbner basis. According to the above proof, this is a consequence of the fact that Buchberger's algorithm runs over the finite number of variables occurring in the generators of $I$.

Another useful grading of $P$ can be introduced in the following way. Consider the set $\hat{\mathbb{N}}=\mathbb{N} \cup\{-\infty\}$ endowed with the binary operations max and + . Then $(\hat{\mathbb{N}}, \max ,+)$ is clearly a commutative idempotent semiring (or commutative dioid or max-plus algebra). Define $\operatorname{deg}: \hat{\Sigma} \rightarrow \hat{\mathbb{N}}$ the mapping such that $\operatorname{deg}(0)=-\infty$ and $\operatorname{deg}(\sigma)=\sum_{i} \alpha_{i}$, for any $\sigma=\prod_{i} \sigma_{i}^{\alpha_{i}}$. Clearly deg is a monoid homomorphism from $(\hat{\Sigma}, \cdot)$ to $(\hat{\mathbb{N}},+)$.

Definition 6.7. Let ord $: M \rightarrow \hat{\mathbb{N}}$ be the unique mapping such that

(i) $\operatorname{ord}(1)=-\infty$;

(ii) $\operatorname{ord}(m n)=\max (\operatorname{ord}(m), \operatorname{ord}(n))$, for any $m, n \in M$;

(iii) $\operatorname{ord}\left(x_{i}(\sigma)\right)=\operatorname{deg}(\sigma)$, for all $i \geq 0$ and $\sigma \in \Sigma$.

Clearly (i),(ii) state that ord is a monoid homomorphism from $(M, \cdot)$ to $(\hat{\mathbb{N}}, \max )$. We call ord the order function of $P$.

For any monomial $m=x_{i_{1}}\left(\delta_{1}\right)^{\alpha_{1}} \cdots x_{i_{k}}\left(\delta_{k}\right)^{\alpha_{k}}$ different from 1 we have that $\operatorname{ord}(m)=\max \left(\operatorname{deg}\left(\delta_{1}\right), \ldots, \operatorname{deg}\left(\delta_{k}\right)\right)$. Clearly, the order function defines a grading $P=\bigoplus_{d \in \hat{\mathbb{N}}} P_{d}$ of the algebra $P$ over the commutative monoid ( $\hat{\mathbb{N}}$, max). Define $P^{(d)}=\bigoplus_{i \leq d} P_{i}=\bigotimes_{\operatorname{deg}(\sigma) \leq d} P(\sigma)$ which is a subalgebra of $P$. Then, if $X$ is a finite set we have that the sequence $\left\{P^{(d)} \mid d \in \hat{\mathbb{N}}\right\}$ is a filtration of $P$ with Noetherian subalgebras where $P^{(0)}=P_{-\infty} \oplus P_{0}$ is isomorphic to $K[X]$.

Definition 6.8. A monomial order $<$ of $\Sigma$ is said to be compatible with deg when $\operatorname{deg}(\sigma)<\operatorname{deg}(\tau)$ implies that $\sigma<\tau$, for any $\sigma, \tau \in \Sigma$.

If $<$ is compatible with deg, note that $<$ is a sequential ordering of $\Sigma$ and $\operatorname{ord}(m)=\operatorname{deg}(\mathrm{w}(m))$, for all $m \in M$. Finally, one has that the weight and order functions clearly coincide when $r=1$.

Proposition 6.9. The order function satisfies the following:

(i) $\operatorname{ord}(\sigma \cdot m)=\operatorname{deg}(\sigma)+\operatorname{ord}(m)$, for any $\sigma \in \Sigma$ and $m \in M$;

(ii) $\operatorname{ord}(\operatorname{lcm}(m, n))=\operatorname{ord}(m n)=\max (\operatorname{ord}(m), \operatorname{ord}(n))$, for all $m, n \in M$. Therefore, if $m \mid n$, then $\operatorname{ord}(m) \leq \operatorname{ord}(n)$.

Proof. For $m=1$ one has $\operatorname{ord}(\sigma \cdot m)=\operatorname{ord}(m)=-\infty=\operatorname{deg}(\sigma)+\operatorname{ord}(m)$. If otherwise $m=x_{i_{1}}\left(\delta_{1}\right)^{\alpha_{1}} \cdots x_{i_{k}}\left(\delta_{k}\right)^{\alpha_{k}}$ then $\sigma \cdot m=x_{i_{1}}\left(\sigma \delta_{1}\right)^{\alpha_{1}} \cdots x_{i_{k}}\left(\sigma \delta_{k}\right)^{\alpha_{k}}$ and hence $\operatorname{ord}(\sigma \cdot m)=\max \left(\operatorname{deg}\left(\sigma \delta_{1}\right), \ldots, \operatorname{deg}\left(\sigma \delta_{k}\right)\right)=\operatorname{deg}(\sigma)+\max \left(\operatorname{deg}\left(\delta_{1}\right), \ldots, \operatorname{deg}\left(\delta_{k}\right)\right)=$ $\operatorname{deg}(\sigma)+\operatorname{ord}(m)$. Property (ii) follows immediately as in Proposition 6.4.

Definition 6.10. Let $I$ be an ideal of $P$. We call $I$ a ord-graded ideal if $I=\sum_{i} I_{i}$ with $I_{i}=I \cap P_{i}$. If $I$ is also a $\Sigma$-ideal then $\sigma \cdot I_{i} \subset I_{\operatorname{deg}(\sigma)+i}$ for any $\sigma \in \Sigma$ and $i \in \hat{\mathbb{N}}$.

Consider now $f, g \in P, f \neq g$ two ord-homogeneous elements. The S-polynomial $h=\operatorname{spoly}(f, g)$ is clearly ord-homogeneous and $\operatorname{ord}(h)=\max (\operatorname{ord}(f), \operatorname{ord}(g))$. 
Then $\operatorname{ord}(f), \operatorname{ord}(g) \leq d$ implies that ord $(h) \leq d$, for some $d \in \mathbb{N}$ and one proves the following result as for Proposition 6.6.

Proposition 6.11 (Truncated termination over the order). Let $I \subset P$ be an ordgraded $\Sigma$-ideal and fix $d \in \mathbb{N}$. Assume $I$ has an ord-homogeneous basis of $H$ such that $H_{d}=\{f \in H \mid \operatorname{ord}(f) \leq d\}$ is a finite set. Then, there is an ord-homogeneous Gröbner $\Sigma$-basis $G \subset I$ such that $G_{d}$ is also a finite set. In other words, if we consider for SigmaGBASIS a selection strategy of the $S$-polynomials based on their orders, we have that the d-truncated version of SIGMAGBASIS terminates in a finite number of steps.

By means of weight and order functions one has criterions, also in the non-graded case, that provide that a Gröbner $\Sigma$-basis is the eventually finite complete one even if it has been computed within some bounded weight or order for the algebra $P$ that is over a finite number of variables. This is of course important because actual computations can be only performed in such a way. As before, we fix a sequential monomial ordering $<$ on $\Sigma$.

Definition 6.12. Let $\prec$ be a monomial $\Sigma$-ordering of $P$. We call $\prec$ compatible with the weight function if $\mathrm{w}(m)<\mathrm{w}(n)$ implies that $m \prec n$, for all $m, n \in M$. In a similar way, one defines when $\prec$ is compatible with the order function.

Proposition 6.13. Let $\prec_{w}$ be a weight $\Sigma$-ordering as in Definition 3.6. Then $\prec_{w}$ is compatible with the weight function. In particular, if the monomial order $<$ of $\Sigma$ is compatible with deg, then $\prec_{w}$ is also compatible with the order function.

Proof. Let $m=m\left(\delta_{1}\right) \cdots m\left(\delta_{k}\right), n=n\left(\delta_{1}\right) \cdots n\left(\delta_{k}\right)$ be two monomials of $P$ with $m\left(\delta_{i}\right), n\left(\delta_{i}\right) \in M\left(\delta_{i}\right)$ and $\delta_{1}>\ldots>\delta_{k}$. Assume $m \prec_{w} n$, that is, $m\left(\delta_{j}\right)=n\left(\delta_{j}\right)$ if $j<i$ and $m\left(\delta_{i}\right) \prec n\left(\delta_{i}\right)$ for some $1 \leq i \leq k$. If $i>1$ or $m\left(\delta_{i}\right) \neq 1$ then clearly $\mathrm{w}(m)=\mathrm{w}(n)=\delta_{1}$. Otherwise, we conclude $\mathrm{w}(m)<\delta_{1}=\mathrm{w}(n)$. Moreover, if $<$ is compatible with deg, then $\operatorname{ord}(m)=\operatorname{deg}(\mathrm{w}(m))<\operatorname{deg}(\mathrm{w}(n))=\operatorname{ord}(n)$ implies that $\mathrm{w}(m)<\mathrm{w}(n)$ and hence $m \prec_{w} n$.

For any $\delta \in \Sigma, d \in \mathbb{N}$ define now $\Sigma_{\delta}=\{\sigma \in \Sigma \mid \sigma \leq \delta\}$ and $\Sigma_{d}=\{\sigma \in \Sigma \mid$ $\operatorname{deg}(\sigma) \leq d\}$.

Proposition 6.14 (Finite $\Sigma$-criterion). Assume the $\Sigma$-ordering of $P$ is compatible with the weight function. Let $G \subset P$ be a finite set and denote $I$ the $\Sigma$-ideal generated by $G$. Moreover, define $\delta=\max _{<}\{\mathrm{w}(\operatorname{lm}(g)) \mid g \in G\}$. Then, $G$ is a Gröbner $\Sigma$-basis of $I$ if and only if for all $f, g \in G$ and for any $\sigma, \tau \in \Sigma$ such that $\operatorname{gcd}(\sigma, \tau)=1$ and $\operatorname{gcd}(\sigma \cdot \operatorname{lm}(f), \tau \cdot \operatorname{lm}(g)) \neq 1$, the S-polynomial $\operatorname{spoly}(\sigma \cdot f, \tau \cdot g)$ has a Gröbner representation with respect to the finite set $\Sigma_{\delta^{2}} \cdot G$. In the same way, if the $\Sigma$-ordering of $P$ is compatible with the order function and $d=\max \{\operatorname{ord}(\operatorname{lm}(g)) \mid$ $g \in G\}$, then $G$ is a Gröbner $\Sigma$-basis of $I$ when the above $S$-polynomials have a Gröbner representation with respect to $\Sigma_{2 d} \cdot G$.

Proof. Let $\operatorname{spoly}(\sigma \cdot f, \tau \cdot g)=h=\sum_{\nu} f_{\nu}\left(\nu \cdot g_{\nu}\right)$ be a Gröbner representation with respect to $\Sigma \cdot G$, that is, $\operatorname{lm}(h) \succeq \operatorname{lm}\left(f_{\nu}\right)\left(\nu \cdot \operatorname{lm}\left(g_{\nu}\right)\right)$ for all $\nu$. We want to bound the elements $\nu \in \Sigma$ with respect to the ordering $<$. Put $m=\operatorname{lm}(f), n=\operatorname{lm}(g)$ and hence $\operatorname{lm}(\sigma \cdot f)=\sigma \cdot m, \operatorname{lm}(\sigma \cdot g)=\sigma \cdot n$. By product criterion, we can assume that $u=\operatorname{gcd}(\sigma \cdot m, \tau \cdot n) \neq 1$. Then there is a variable $x_{i}(\sigma \alpha)=x_{i}(\tau \beta)$ that divides $u$ where $x_{i}(\alpha)$ divides $m$ and hence $\alpha \leq \mathrm{w}(m) \leq \delta$ and $x_{i}(\beta)$ divides $n$ and therefore $\beta \leq \mathrm{w}(n) \leq \delta$. Then $\sigma \alpha=\tau \beta$ and one has that $\sigma|\beta, \tau| \alpha$ 
because $\operatorname{gcd}(\sigma, \tau)=1$. We conclude that $\sigma, \tau \leq \delta$ and if $v=\operatorname{lcm}(\sigma \cdot m, \tau \cdot m)$ then $\mathrm{w}(v)=\max (\sigma \mathrm{w}(m), \tau \mathrm{w}(n)) \leq \delta^{2}$. Clearly $v \succ \operatorname{lm}(h) \succeq \nu \cdot \operatorname{lm}\left(g_{\nu}\right)$ and hence $\delta^{2} \geq \mathrm{w}(v) \geq \nu \mathrm{w}\left(\operatorname{lm}\left(g_{\nu}\right)\right) \geq \nu$. In a similar way, one argues for the order function.

The above criterion implies that with respect to $\Sigma$-orderings compatible with weight or order functions one has an algorithm able to compute a finite Gröbner $\Sigma$ basis, whenever this exists, in a finite number of steps. To fix ideas, let us consider only weights. If $G$ is a finite $\Sigma$-basis of $I$ and $\delta=\max _{<}\{\mathrm{w}(\operatorname{lm}(g)) \mid g \in G\}$, we may start considering all S-polynomials $\operatorname{spoly}(\sigma \cdot f, \tau \cdot g)$ with $f, g \in G$ and $\sigma, \tau \in \Sigma$ such that $\operatorname{gcd}(\sigma, \tau)=1$ and $\operatorname{gcd}(\sigma \cdot \operatorname{lm}(f), \tau \cdot \operatorname{lm}(g)) \neq 1$. Note that such S-polynomials are in a finite number since in the above proof we observed that $\sigma, \tau \leq \delta$ and the monomial ordering of $\Sigma$ is sequential. Moreover, one also has that these S-polynomials can be reduced only by elements of the finite set $\Sigma_{\delta^{2}} \cdot G$. If, as a result of some reduction, a new element $f \neq 0$ has to be added to the $\Sigma$-basis $G$ and $\mathrm{w}(\operatorname{lm}(f))=\delta^{\prime}>\delta$, then it is sufficient to update the weight bound $\delta$ to $\delta^{\prime}$.

\section{Homogenizing With RESPECT TO ORDER FUNCTION}

The purpose of this section is to analyze (de)homogenization processes in the context of $\Sigma$-ideals. Such methods are generally developed to have structures and computations that are homogeneous with respect to some grading, even if the input data are not as such. Besides to the theoretical advantages as the concept of projective closure, these techniques usually imply computational benefits (see for instance [3]). Note that for $\Sigma$-ideals it is completely useless to consider classical gradings (total degree, multidegree, etc.) since they do not provide compatibility conditions with the $\Sigma$-action like the ones contained in Proposition 6.4 and Proposition 6.9. We decided then to present (de)homogenization methods only for the grading defined by the order function because univariate homogenizations are usually more efficient than multivariate ones since leading monomials are preserved by the homogenization process. Note finally that a major difference of the theory we present here with the classical one is that the kernel of the dehomogenizing homomorphism contains a non-trivial graded ideal which implies that the homogenization process has to be considered modulo such ideal.

Let $t$ be a new variable disjoint with $X$. Define $\bar{X}=X \cup\{t\}, \bar{X}(\Sigma)=\bar{X} \times$ $\Sigma, \bar{P}=K[\bar{X}(\Sigma)]$ and finally $\bar{M}=\operatorname{Mon}(\bar{P})$. Consider the algebra endomorphism $\varphi: \bar{P} \rightarrow \bar{P}$ such that $x_{i}(\sigma) \mapsto x_{i}(\sigma)$ and $t(\sigma) \mapsto 1$, for all $i, \sigma$. Clearly $\varphi^{2}=\varphi$ and $P=\varphi(\bar{P})$. Moreover, one has that $\varphi$ is a $\Sigma$-algebra endomorphism. Then $\varphi$ defines a bijective correspondence between all $\Sigma$-ideals of $P$ and $\Sigma$-ideals of $\bar{P}$ containing $\operatorname{ker} \varphi=\langle t(1)-1\rangle_{\Sigma}$.

Definition 7.1. Denote by $N=N_{\text {ord }}$ the largest ord-graded $\Sigma$-ideal contained in $\operatorname{ker} \varphi$, that is, the ideal generated by all ord-homogeneous elements $f \in \bar{P}$ such that $\varphi(f)=0$.

Proposition 7.2. The ideal $N \subset \bar{P}$ is generated by the elements

(i) $t(\sigma)-t(\tau)$ for all $\sigma, \tau \in \Sigma, \sigma \neq \tau, \operatorname{deg}(\sigma)=\operatorname{deg}(\tau)$;

(ii) $t(\sigma) t(\tau)-t(\sigma), x(\sigma) t(\tau)-x(\sigma)$ for any $\sigma, \tau \in \Sigma, \operatorname{deg}(\sigma) \geq \operatorname{deg}(\tau)$.

Proof. Let $f \in \bar{P}$ be a ord-homogeneous element such that $\varphi(f)=0$. Since the polynomials of types (i) and (ii) clearly belong to $N$, we have to prove that $f$ is 
congruent to 0 modulo (i),(ii). Assume first that all variables of $f$ belong to $t(\Sigma)=$ $\{t(\sigma) \mid \sigma \in \Sigma\}$. Recall that if $m=t\left(\delta_{1}\right)^{\alpha_{1}} \cdots t\left(\delta_{k}\right)^{\alpha_{k}}$ is any monomial of $f$ then $d=\operatorname{ord}(f)=\max \left(\operatorname{deg}\left(\delta_{1}\right), \ldots, \operatorname{deg}\left(\delta_{k}\right)\right)$. Therefore, one has that $f$ is congruent modulo (ii) to $f^{\prime}=\sum_{i} c_{i} t\left(\tau_{i}\right)$ where $\tau_{i} \in \Sigma, \operatorname{deg}\left(\tau_{i}\right)=d$ and $c_{i} \in K, \sum_{i} c_{i}=0$. By applying identity (i) it follows that $f$ is congruent to $\left(\sum_{i} c_{i}\right) t(\sigma)=0$ for some fixed $\sigma$ such that $\operatorname{deg}(\sigma)=d$.

Consider now the general case when the variables of $f$ belong to $\bar{X}(\Sigma)$. Fix $\sigma \in \Sigma$ such that $\operatorname{deg}(\sigma)=d$. Modulo the identities (i),(ii), one has that $f$ is congruent to a polynomial $f^{\prime}$ whose monomials are either of type $m \in M$ such that $\operatorname{ord}(m)=d$ or of type $t(\sigma) n$ where $n \in M$, ord $(n)<d$. We show that in fact $f^{\prime}=0$. Denote $f^{\prime}=t(\sigma) g-h$ where $g, h$ are polynomials in $P, h$ is ord-homogeneous and $\operatorname{ord}(h)=d$. Since $0=\varphi\left(f^{\prime}\right)=g-h$ one has that $f^{\prime}=(t(\sigma)-1) g$. If we assume $g \neq 0$ then the monomials $n$ of $g$ are such that $\operatorname{ord}(n)=d$ which is a contradiction.

Now, we want to define a bijective correspondence between all $\Sigma$-ideals of $P$ and some class of ord-graded $\Sigma$-ideals of $\bar{P}$ containing $N$.

Definition 7.3. Let $I$ be any $\Sigma$-ideal of $P$. We define $I^{*} \subset \bar{P}$ the largest ordgraded $\Sigma$-ideal contained in the preimage $\varphi^{-1}(I)$, that is, $I^{*}$ is the ideal generated by all ord-homogeneous elements in $\varphi^{-1}(I)$. Clearly $N=0^{*} \subset I^{*}$. We call $I^{*}$ the ord-homogenization of the $\Sigma$-ideal $I$.

Definition 7.4. Let $f \in P, f \neq 0$ and denote $f=\sum_{d} f_{d}$ the decomposition of $f$ in its ord-homogeneous components. We define topord $(f)=d^{\prime}=\max \{d\}$. If $f \in K$, that is, $d^{\prime}=-\infty$, we put $f^{*}=f$. Otherwise, we denote $f^{*}=t(\sigma) f$ where $\sigma \in \Sigma \operatorname{such}$ that $\operatorname{deg}(\sigma)=d^{\prime}$. We call topord $(f)$ the top order of $f$ and $f^{*}$ its ord-homogenization.

Note that $\varphi\left(f^{*}\right)=f$ and hence the element $f^{*}$ is essentially defined modulo the ideal $N$ (see also the next result). Owing to generators (i) of $N$ in Proposition 7.2, all variables $t(\sigma)$ such that $\operatorname{deg}(\sigma)=d^{\prime}$ are congruent modulo $N$. Then we do not need to specify which of these variables we use for defining $f^{*}=t(\sigma) f$.

Proposition 7.5. Let $I$ be a $\Sigma$-ideal of $P$. Then $I^{*}=\left\langle f^{*} \mid f \in I, f \neq 0\right\rangle+N$.

Proof. Denote $J=\left\langle f^{*} \mid f \in I\right\rangle+N$. Clearly $J \subset I^{*}$. Let $g \in I^{*}$ be an ordhomogeneous element and define $f=\varphi(g) \in I$. If $f=0$ then $g \in N \subset J$. Otherwise, denote $d=\operatorname{topord}(f)$ and $d^{\prime}=\operatorname{ord}(g)$. Since clearly $d^{\prime} \geq d$ one has that $g$ is congruent modulo $N$ to $h=t(\sigma) f$, where $\sigma \in \Sigma$ such that $\operatorname{deg}(\sigma)=d^{\prime}$. Hence, if $d^{\prime}=d$, then $h$ is congruent exactly to $f^{*}$. Otherwise, the polynomial $h$ is congruent to $t(\sigma) f^{*}$. In both cases, we conclude that $g$ is congruent modulo $N \subset J$ to an element of $J$ and therefore $g \in J$.

If $I \subset P$ is a $\Sigma$-ideal one clearly has that $\varphi\left(I^{*}\right)=I$. Moreover, if $J \subset \bar{P}$ is an ord-graded $\Sigma$-ideal containing $N$, then in general $J \subset \varphi(J)^{*}$.

Definition 7.6. Let $N \subset J \subset \bar{P}$ be an ord-graded $\Sigma$-ideal. Define $J^{\prime}=\varphi(J)^{*}=$ $\left\langle\varphi(f)^{*}\right| f \in J, f \notin N, f$ ord-homogeneous $\rangle+N$. Then $J \subset J^{\prime} \subset \bar{P}$ is an ord-graded $\Sigma$-ideal that we call the saturation of $J$. 
Definition 7.7. Let $J \subset \bar{P}$ be an ord-graded $\Sigma$-ideal containing $N$. We say that $J$ is saturated if $J$ coincides with its saturation $\varphi(J)^{*}$, that is, for any ordhomogeneous element $f \in J, f \notin N$ one has that $\varphi(f)^{*} \in J$. If $I$ is a $\Sigma$-ideal of $P$ then its ord-homogenization $I^{*}$ is clearly a saturated ideal.

Therefore, a bijective correspondence is given between all $\Sigma$-ideals of $P$ and the saturated ord-graded $\Sigma$-ideals of $\bar{P}$ containing $N$.

Now, we want to analyze the behaviour of Gröbner $\Sigma$-bases under homogenization and dehomogenization. Note that the arguments of Proposition 7.2 imply clearly that the polynomials (i) and (ii) are in fact Gröbner bases of the ideal $N$ with respect to any monomial ordering of $\bar{P}$. For this reason we introduce the following notion.

Definition 7.8. A monomial $m \in \bar{M}$ is said to be normal modulo $N$ if $m \in M$ or $m=t(\sigma) n$ with $n \in M, \sigma \in \Sigma$ such that $d=\operatorname{deg}(\sigma)>\operatorname{ord}(n)$. Moreover, we require that $t(\sigma)=\min _{\prec}\{t(\tau) \mid \operatorname{deg}(\tau)=d\}$. A polynomial $f \in \bar{P}$ is in normal form modulo $N$ if all its monomials are normal modulo $N$.

Note that owing to generators (i) of the ideal $N$, we choose $t(\sigma)=\min _{\prec}\{t(\tau) \mid$ $\operatorname{deg}(\tau)=d\}$ since in this case $\operatorname{lm}(t(\sigma)-t(\tau))=t(\tau)$.

Definition 7.9. Let $\prec$ be a $\Sigma$-ordering of $\bar{P}$ compatible with the order function. We call $\prec$ a ord-homogenization $\Sigma$-ordering if $t(\sigma) m \prec n$ for all $m, n \in M, \sigma \in \Sigma$ such that $\operatorname{deg}(\sigma)=\operatorname{ord}(n)>\operatorname{ord}(m)$.

It is easy to define one of the above orderings. Fix, for instance, the lex or degrevlex monomial order on the polynomial $\operatorname{ring} \bar{P}(1)=K\left[x_{0}(1), x_{1}(1), \ldots, t(1)\right]$ where $x_{0}(1) \succ x_{1}(1) \succ \ldots \succ t(1)$. Moreover, fix a monomial ordering on $\Sigma$ which is compatible with deg and define the weight $\Sigma$-ordering $\prec_{w}$ of $\bar{P}$ as in Definition 3.6. Clearly $\prec_{w}$ is a ord-homogenization $\Sigma$-ordering.

From now on, we assume $\bar{P}$ be endowed with an ord-homogenization $\Sigma$-ordering.

Proposition 7.10. Let $p, q \in \bar{M}$ be two normal monomials modulo $N$ such that $\operatorname{ord}(p)=\operatorname{ord}(q)$. Then $p \prec q$ implies that $\varphi(p) \prec \varphi(q)$.

Proof. By definition, the monomials $p, q$ are of type $m \in M$ or $t(\sigma) m$ with $\operatorname{deg}(\sigma)>$ $\operatorname{ord}(m)$. Since $\prec$ is an ord-homogenization order, when comparing two such monomials of the same order one has only the following cases: $m \prec n, t(\sigma) m \prec t(\sigma) n$ or $t(\sigma) m \prec n$. Then, we have to prove $\varphi(p)=m \prec n=\varphi(q)$ only when $t(\sigma) m \prec n$. This follows immediately from $\prec$ is compatible with the order function and $\operatorname{ord}(m)<\operatorname{ord}(n)=\operatorname{deg}(\sigma)$.

From now on, for any $f \in P, f \neq 0$ we denote by $f^{*}$ the normal form of $t(\sigma) f$ modulo $N$ where $\sigma \in \Sigma, \operatorname{deg}(\sigma)=\operatorname{topord}(f)$.

Proposition 7.11. Let $f \in \bar{P}, f \neq 0$ be an ord-homogeneous polynomial in normal form modulo $N$. Then $\operatorname{lm}(\varphi(f))=\varphi(\operatorname{lm}(f))$. Moreover, we have that $\operatorname{lm}\left(f^{*}\right)=$ $\operatorname{lm}(f)$ for all $f \in P, f \neq 0$.

Proof. The first part of the statement follows immediately from Proposition 7.10 , Moreover, if $\sigma \in \Sigma, \operatorname{deg}(\sigma)=\operatorname{topord}(f)$, then $t(\sigma)$ cannot appear in the leading monomial of $f^{*}$ and hence $\operatorname{lm}\left(f^{*}\right)=\operatorname{lm}(f)$. 
Definition 7.12. Let $N \subset J \subset \bar{P}$ be a $\Sigma$-ideal. Moreover, let $G \subset J$ be a subset of polynomials in normal form modulo $N$. We say that $G$ is a Gröbner $\Sigma$-basis of $J$ modulo $N$ if $G \cup N$ is a Gröbner $\Sigma$-basis of $J$.

Proposition 7.13. Let $N \subset J \subset \bar{P}$ be a ord-graded $\Sigma$-ideal. If $G$ is an ordhomogeneous Gröbner $\Sigma$-basis of $J$ modulo $N$ then $\varphi(G)$ is a Gröbner $\Sigma$-basis of $\varphi(J)$.

Proof. Since $G$ is a Gröbner $\Sigma$-basis of $J$ modulo $N$ we have that for any ordhomogeneous polynomial $f \in J, f \neq 0$ in normal form modulo $N$ there is an element $g \in G$ and $\sigma \in \Sigma$ such that $\sigma \cdot \operatorname{lm}(g) \mid \operatorname{lm}(f)$. Then, by applying the $\Sigma$-algebra endomorphism $\varphi$ one obtains that $\sigma \cdot \operatorname{lm}(\varphi(g)) \mid \operatorname{lm}(\varphi(f))$, that is, $\varphi(G)$ is a Gröbner $\Sigma$-basis of $\varphi(J)$.

Proposition 7.14. Let $I \subset P$ be a $\Sigma$-ideal and let $G$ be a Gröbner $\Sigma$-basis of $I$. Then $G^{*}=\left\{g^{*} \mid g \in G\right\}$ is an ord-homogeneous Gröbner basis of $I^{*}$ modulo $N$. Moreover, one has that $\operatorname{lm}\left(G^{*}\right)=\operatorname{lm}(G)$.

Proof. Let $f^{\prime} \in I^{*}$ be an ord-homogeneous element in normal form modulo $N$ and put $f=\varphi\left(f^{\prime}\right) \in I$. Then, either $f^{\prime}=f^{*}$ or $f^{\prime}=t(\sigma) f^{*}$ with $\operatorname{ord}\left(f^{\prime}\right)=\operatorname{deg}(\sigma)>$ $\operatorname{ord}\left(f^{*}\right)=\operatorname{topord}(f)$. Since $G$ is a Gröbner $\Sigma$-basis of $I$ there is $g \in G, \tau \in \Sigma$ such that $\tau \cdot \operatorname{lm}(g) \mid \operatorname{lm}(f)$. By Proposition 7.11 one has that $\operatorname{lm}(f)=\operatorname{lm}\left(f^{*}\right)$ and $\operatorname{lm}(g)=\operatorname{lm}\left(g^{*}\right)$. Therefore, $\tau \cdot \operatorname{lm}\left(g^{*}\right)$ divides $\operatorname{lm}\left(f^{*}\right)$ and this monomial clearly divides $\operatorname{lm}\left(f^{\prime}\right)$.

By the above propositions we obtain immediately what follows.

Corollary 7.15. Let $N \subset J \subset \bar{P}$ be an ord-graded $\Sigma$-ideal and denote $J^{\prime}=\varphi(J)^{*}$ its saturation. Moreover, let $G$ be an ord-homogeneous Gröbner $\Sigma$-basis of $J$ modulo $N$. Then $G^{\prime}=\varphi(G)^{*}=\left\{\varphi(g)^{*} \mid g \in G\right\}$ is an ord-homogeneous Gröbner $\Sigma$-basis of $J^{\prime}$ modulo $N$. Moreover, we have $\operatorname{lm}\left(G^{\prime}\right)=\operatorname{lm}(\varphi(G))$.

Let $I \subset P$ be any $\Sigma$-ideal. The previous results suggest an alternative method to calculate a Gröbner $\Sigma$-basis of $I$ which is based only on ord-homogeneous computations. Assume $H$ is any $\Sigma$-basis of $I$ and denote as before $H^{*}=\left\{f^{*} \mid f \in H\right\}$. Clearly $J=\left\langle H^{*}\right\rangle_{\Sigma}+N$ is an ord-homogeneous $\Sigma$-ideal of $\bar{P}$ containing $N$ such that $\varphi(J)=I$. Assume now we compute $G$ an ord-homogeneous Gröbner $\Sigma$-basis of $J$ modulo $N$. Then, $\varphi(G)$ is a Gröbner $\Sigma$-basis of $I$. Note that by using an ord-based selection strategy for the S-polynomials, the Gröbner $\Sigma$-basis $G$ can be obtained order by order automatically minimal, that is, $\sigma \cdot \operatorname{lm}(f)$ does not divide $\operatorname{lm}(g)$ for all $f, g \in G, f \neq g$ and $\sigma \in \Sigma$. This is clearly a computational advantage, but since generally $\operatorname{lm}(G) \neq \operatorname{lm}(\varphi(G))$ one has that $\varphi(G)$ may be not minimal. In the worst case, the ideal $J$ may have an infinite and hence uncomputable minimal Gröbner $\Sigma$-basis but $I$ has a finite one. This is clearly not the case when one considers a saturated ideal $J^{\prime}=I^{*}$ since we have $\operatorname{lm}\left(G^{\prime}\right)=\operatorname{lm}\left(\varphi\left(G^{\prime}\right)\right)$ when $G^{\prime}$ is a minimal Gröbner $\Sigma$-basis of $J^{\prime}$. Note that this nice property depends on the fact that we deal with univariate homogenization. A drawback is that if one computes the saturation $J^{\prime}$ by means of the ideal $J$, according to Corollary 17.15, one again has to compute a Gröbner $\Sigma$-basis of $J$. Then, a better approach consists in computing "on the fly" the Gröbner $\Sigma$-basis of $J^{\prime}$ starting from the generating set $\left\{f^{*} \mid f \in H\right\}$. In other words, any time that a new generator $g$ of the ord-homogeneous Gröbner $\Sigma$-basis arises from the reduction of an S-polynomial, we saturate $g$; that is, we 
substitute this polynomial with $\varphi(g)^{*}$. In formal terms, the algorithm one obtains is the following one.

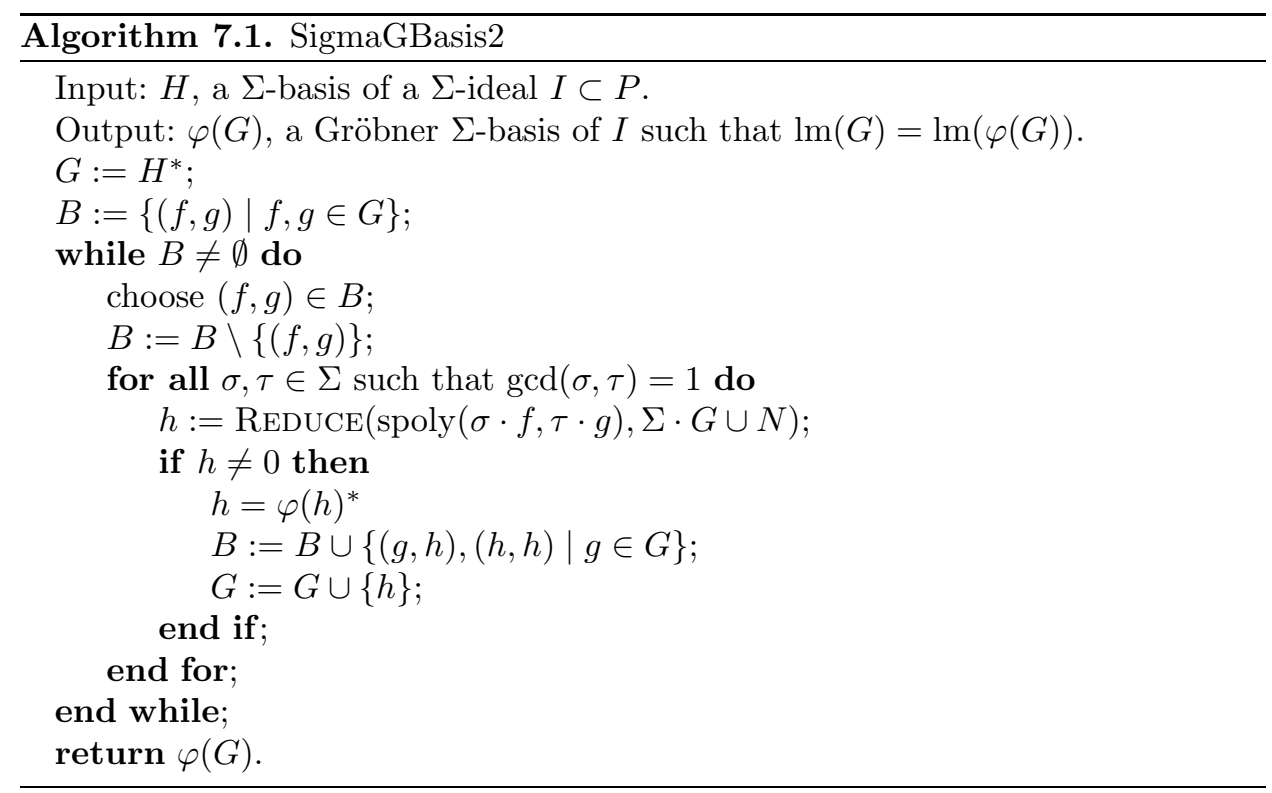

Proposition 7.16. The algorithm SigmaGBASIS2 is correct.

Proof. Note that at each step we are inside an ideal $J$ such that $\varphi(J)=I$, that is, whose saturation is $J^{\prime}=I^{*}$. Moreover, for any ord-homogeneous element $h \in \bar{P}$ which is in normal form modulo $N$ one has that $h^{\prime}=\varphi(h)^{*}$ divides $h$. This implies that if an S-polynomial is reduced to zero by adding $h$ to the basis $G$, the same holds true if we substitute $h$ with $h^{\prime}$. In case of termination, owing to the set $G$ is an ord-homogeneous Gröbner $\Sigma$-basis of $J$ modulo $N$ whose elements are all saturated, by Corollary 7.15 we may conclude that $J=J^{\prime}$ and hence $\varphi(G)$ is a Gröbner $\Sigma$-basis of $I$ such that $\operatorname{lm}(G)=\operatorname{lm}(\varphi(G))$.

About termination or just termination up to some order $d$, this is not provided in general for the above algorithm. The reason is that even if all computations are ord-homogeneous, because of the saturation $h=\varphi(h)^{*}$ that may decrease the order we cannot be sure at some suitable step that we will not get additional elements of order $\leq d$ in the steps that follow.

\section{An illustrative example (CONTinued)}

We now apply the algorithm SigmaGBAsis2 to the same $\Sigma$-ideal that has been considered in Section 5 for illustrating SigmaGBAsis. Recall that this ideal is $I=\left\langle g_{1}, g_{2}\right\rangle \subset P=K[X(\Sigma)]$ where $X=\{x, y\}, \Sigma=\mathbb{N}^{2}$ and

$$
\begin{aligned}
& g_{1}=y(1,1) y(1,0)-2 x(0,1)^{2}, \\
& g_{2}=y(2,0)+x(0,0) x(1,0) .
\end{aligned}
$$

Now let $\bar{X}=\{x, y, t\}$ and define the polynomial algebra $\bar{P}=K[\bar{X}(\Sigma)]$ with variables $x(i, j), y(i, j), t(i, j)$, for all $i, j \geq 0$. We consider the $\Sigma$-algebra endomorphism 
$\varphi: \bar{P} \rightarrow \bar{P}$ such that $x(i, j) \mapsto x(i, j), y(i, j) \mapsto y(i, j)$ and $t(i, j) \mapsto 1$. In Proposition 7.2 we proved that the largest ord-graded $\Sigma$-ideal contained in $\operatorname{ker} \varphi$ is the ideal $N \subset \bar{P}$ generated by the polynomials

$$
\begin{aligned}
& t(i, j)-t(k, l) \text { where }(i, j) \neq(k, l), i+j=k+l \\
& t(i, j) t(k, l)-t(i, j), x(i, j) t(k, l)-x(i, j) \text { where } i+j \geq k+l .
\end{aligned}
$$

Moreover, we define an ord-homogenization $\Sigma$-ordering of $\bar{P}$ (Definition 17.9) as the weight $\Sigma$-ordering given by the degrevlex ordering of $\Sigma\left(\sigma_{1}>\sigma_{2}\right)$ and the lex monomial ordering of $K[x(0,0), y(0,0), t(0,0)](x(0,0) \succ y(0,0) \succ t(0,0))$. Note that such a $\Sigma$-ordering extends the one defined for $P \subset \bar{P}$ in Section 5 . In practice, it is the lexicographic monomial ordering of $\bar{P}$ such that

$$
\begin{aligned}
& \ldots \succ x(2,0) \succ y(2,0) \succ t(2,0) \succ x(1,1) \succ y(1,1) \succ t(1,1) \succ x(0,2) \\
& \succ y(0,2) \succ t(0,2) \succ x(1,0) \succ y(1,0) \succ t(1,0) \succ x(0,1) \succ y(0,1) \succ t(0,1) \\
& \succ x(0,0) \succ y(0,0) \succ t(0,0) .
\end{aligned}
$$

Recall that a Gröbner $\Sigma$-basis of $I$ with respect to such ordering is given by the elements $g_{1}, g_{2}$ together with

$$
\begin{aligned}
& g_{3}=y(1,2) x(0,1)^{2}-y(1,0) x(0,2)^{2}, \\
& g_{4}=2 x(1,1)^{2}-x(0,0) x(1,0) x(0,1) x(1,1) .
\end{aligned}
$$

We then introduce ord-homogenizations of the polynomials $g_{1}, g_{2}$ that are

$$
\begin{aligned}
& g_{1}^{*}=y(1,1) y(1,0)-2 t(0,2) x(0,1)^{2}, \\
& g_{2}^{*}=y(2,0)+t(0,2) x(0,0) x(1,0) .
\end{aligned}
$$

We start applying SigmaGBAsis2 to $G=\left\{g_{1}^{*}, g_{2}^{*}\right\}$ by considering the S-polynomials

$$
\begin{aligned}
& s_{1}=\operatorname{spoly}\left((0,1) \cdot g_{1}^{*}, g_{1}^{*}\right), \\
& s_{2}=\operatorname{spoly}\left((1,0) \cdot g_{1}^{*}, g_{2}^{*}\right), \\
& s_{3}=\operatorname{spoly}\left((1,0) \cdot g_{1}^{*},(0,1) \cdot g_{2}^{*}\right) .
\end{aligned}
$$

By reducing $s_{1}$ with respect to $\Sigma \cdot G \cup N$ one obtains exactly the polynomial

$$
g_{3}^{*}=y(1,2) x(0,1)^{2}-t(0,3) y(1,0) x(0,2)^{2} .
$$

Clearly $g_{3}^{*}$ is already a saturated element. Then $G=\left\{g_{1}^{*}, g_{2}^{*}, g_{3}^{*}\right\}$ and we form the new S-polynomials

$$
\begin{aligned}
& s_{4}=\operatorname{spoly}\left((0,1) \cdot g_{1}^{*}, g_{3}^{*}\right), \\
& s_{5}=\operatorname{spoly}\left((0,2) \cdot g_{1}^{*}, g_{3}^{*}\right), \\
& s_{6}=\operatorname{spoly}\left((0,2) \cdot g_{2}^{*},(1,0) \cdot g_{3}^{*}\right) .
\end{aligned}
$$

With respect to $\Sigma \cdot G \cup N$, one has the reductions $s_{4} \rightarrow 0$ and $s_{2} \rightarrow h$ where

$$
h=2 t(0,3) x(1,1)^{2}-t(0,3) x(0,0) x(1,0) x(0,1) x(1,1)=t(0,3) g_{4}^{*} .
$$

By saturating this element as $\varphi(h)^{*}=g_{4}^{*}$, we update $G=\left\{g_{1}^{*}, g_{2}^{*}, g_{3}^{*}, g_{4}^{*}\right\}$ and another S-polynomial is defined as

$$
s_{7}=\operatorname{spoly}\left((1,0) \cdot g_{3}^{*}, g_{4}^{*}\right) .
$$

All remaining S-polynomials $s_{3}, s_{5}, s_{6}, s_{7}$ reduce to zero with respect to $\Sigma \cdot G \cup N$ and we conclude that $\varphi(G)=\left\{g_{1}, g_{2}, g_{3}, g_{4}\right\}$ is a Gröbner $\Sigma$-basis of $I \subset P$. 


\section{Testing AND TIMINGS}

In this section we present a set of tests for the algorithms SigmaGBASIS and SIgmaGBASIs2 which is based on an experimental implementation of them in the language of Maple. This is actually the first implementation of algorithms for computing Gröbner bases of systems of linear or non-linear partial difference equations. Note that for the linear case one has the packages LDA (Linear Difference Algebra) 12 and Ore_algebra[shift_algebra] in the Maple distribution. The main idea we followed when coding the proposed algorithms is that they can be considered variants of the classical Buchberger's algorithm where some of the computations can be avoided by means of the symmetry defined by the monoid $\Sigma$. In fact, as explained in the previous sections, a "basic" approach to calculate a Gröbner $\Sigma$-basis of a $\Sigma$-ideal $I$ generated by a $\Sigma$-basis $H$ consists in applying Buchberger's algorithm to the basis $\Sigma \cdot H$. One therefore obtains a Gröbner basis $G^{\prime}$ of $I$ from which a Gröbner $\Sigma$-basis $G \subset G^{\prime}$ can be extracted such that $\Sigma \cdot \operatorname{lm}(G)=\operatorname{lm}\left(G^{\prime}\right)$. Clearly, chain and coprime criterions can be used in the usual way in the procedure. Then, the algorithm SIGMAGBASIS can be understood as the variant that prescribes the application also of the $\Sigma$-criterion (Proposition 4.7) to the S-polynomials spoly $(\sigma \cdot f, \tau \cdot g)$ and to add the set of all shifts $\Sigma \cdot h$ to the current basis when a new element $h$ arises from the reduction of an S-polynomial. Then, the Gröbner $\Sigma$-basis of $I$ is simply the union of the initial basis $H$ with the new elements $h$. Recall that the procedure is correct only if one uses a monomial $\Sigma$-ordering. Clearly, from the set $\Sigma$ is infinite, it follows that actual computations can be only performed with a finite subset of $\Sigma$ that is over a finite set of variables of $P=K[X(\Sigma)]$. Typically, one fixes a bound $d$ for the degree of the elements of $\Sigma$ that is for the order of the variables $x_{i}(\sigma)$. Owing to the finite $\Sigma$-criterion (Proposition 6.14), a basis obtained with a monomial ordering compatible with the order function is certified to be a complete Gröbner $\Sigma$-basis if the order bound is at least the double of the maximum top order of its elements.

In addition to the basic procedure for the computation of Gröbner $\Sigma$-bases and the algorithm SigmaGBASIS, for the experiments we consider also a variant of the latter method where the $\Sigma$-criterion is suppressed but one continues to shift the reduced form of the S-polynomials. This procedure is tested to the aim of understanding the contribution of any of the implemented strategies. Finally, we propose an implementation of the algorithm SigmaGBAsis2 based on the saturation of a $\Sigma$-ideal with respect to the grading defined by the order function. In practice, once one has homogenized the initial generators, the saturation $\varphi(h)^{*}$ is performed before the application of shifting, for each new element $h$ obtained by the reduction of an S-polynomial. In output one returns the dehomogenization of the computed basis. Note that this procedure is correct only if one uses a $\Sigma$-ordering which is compatible with the order function and if the polynomials are kept in normal form modulo $N$ during the computations.

The monomial $\Sigma$-orderings of $P$ that we consider for the tests are defined in the following way. One initially has to fix a monomial ordering for $\Sigma$ and we choose degrevlex in order to provide compatibility with the degree. Then, one fixes a monomial ordering, for us lex, over the subring $P(1)=K[X(1)]$ or $P\left(x_{0}\right)=$ $K\left[x_{0}(\Sigma)\right]$ that is extended as a block ordering to the polynomial ring $P=K[X(\Sigma)]$ according to the choice of a variables ranking based on weight or index respectively. For a detailed description of these orderings in the considered examples see the Appendix. In the table of tests, we distinguish weight or index ranking by the 
letters "w" and "i". The integer that comes before these letters refers to the fixed order bound. Note that the algorithm SigmaGBAsis2 is compatible only with rankings of type weight.

For the basic variant of the Gröbner $\Sigma$-bases algorithm, one can clearly use any implementation of Buchberger's algorithm as, for instance, the one contained in the package Groebner of Maple. We have preferred instead to develop all different variants in order to have the same implementation and hence the same efficiency, for the fundamental subroutines of the algorithms. In this way, for the basic version we have been also able to access to important parameters of the computation as the total number of S-polynomial reductions. This number is for us the sum of the actual S-polynomials with the initial generators that are interreduced. Note that our implementation of Buchberger's algorithm is in fact generally comparable with the built-in ones of Maple. For instance, the test falkow-6w-basic takes 9 hours, but using Groebner[Basis] it takes 11 hours with method=buchberger and 8.5 hours with method=maplef4. Other parameters that are considered for the experiments are the number of input and output generators. Note that for the basic algorithm we count generators and not $\Sigma$-generators. Finally, the parameter "minout" refers to the number of elements of a minimal Gröbner $\Sigma$-basis. All examples have been computed with Maple 12 running on a server with a four core Intel Xeon at $3.16 \mathrm{GHz}$ and 64 GB RAM. The timings are given in hour-minute-second format.

\begin{tabular}{|l|c|c|c|c|c|}
\hline Example & in & out & minout & pairs & time \\
\hline falkow-6w-sigma & 4 & 5 & 5 & 5 & $18 \mathrm{~s}$ \\
falkow-6w-nocrit & 4 & 5 & 5 & 8 & $57 \mathrm{~m} 1 \mathrm{~s}$ \\
falkow-6w-sigma2 & 4 & 5 & 5 & 5 & $61 \mathrm{~s}$ \\
falkow-6w-basic & 157 & 157 & 5 & 157 & $9 \mathrm{~h} 8 \mathrm{~m} 11 \mathrm{~s}$ \\
\hline falkow-6i-sigma & 4 & 10 & 9 & 25 & $1 \mathrm{~m} 45 \mathrm{~s}$ \\
falkow-6i-nocrit & 4 & 10 & 9 & 34 & $1 \mathrm{~m} 53 \mathrm{~s}$ \\
falkow-6i-basic & 157 & 163 & 9 & 172 & $1 \mathrm{~m} 44 \mathrm{~s}$ \\
\hline navier-8w-sigma & 4 & 6 & 5 & 9 & $26 \mathrm{~s}$ \\
navier-8w-nocrit & 4 & 6 & 5 & 22 & $3 \mathrm{~h} 46 \mathrm{~m} 29 \mathrm{~s}$ \\
navier-8w-sigma2 & 4 & 6 & 5 & 9 & $6 \mathrm{~m} 4 \mathrm{~s}$ \\
navier-8w-basic & 86 & - & - & - & $>3$ days \\
\hline navier-8i-sigma & 4 & 9 & 4 & 15 & $12 \mathrm{~s}$ \\
navier-8i-nocrit & 4 & 9 & 4 & 37 & $16 \mathrm{~s}$ \\
navier-8i-basic & 86 & 86 & 4 & 86 & $10 \mathrm{~s}$ \\
\hline heat-12w-sigma & 5 & 5 & 5 & 7 & $1 \mathrm{~m} 10 \mathrm{~s}$ \\
heat-12w-nocrit & 5 & 5 & 5 & 137 & $1 \mathrm{~m} 46 \mathrm{~s}$ \\
heat-12w-sigma2 & 5 & 5 & 5 & 7 & $2 \mathrm{~m} 15 \mathrm{~s}$ \\
heat-12w-basic & 378 & 246 & 5 & 378 & $1 \mathrm{~m} 33 \mathrm{~s}$ \\
\hline eq26-12w-sigma & 1 & 43 & 28 & 557 & $2 \mathrm{~m} 4 \mathrm{~s}$ \\
eq26-12w-nocrit & 1 & 43 & 28 & 790 & $1 \mathrm{~m} 50 \mathrm{~s}$ \\
eq26-12w-sigma2 & 1 & 43 & 28 & 557 & $24 \mathrm{~m} 33 \mathrm{~s}$ \\
eq26-12w-basic & 10 & 208 & 28 & 1673 & $6 \mathrm{~m} 40 \mathrm{~s}$ \\
\hline eq27-12w-sigma & 1 & 28 & 18 & 609 & $14 \mathrm{~s}$ \\
eq27-12w-nocrit & 1 & 28 & 18 & 923 & $22 \mathrm{~s}$ \\
eq27-12w-sigma2 & 1 & 28 & 18 & 609 & $25 \mathrm{~s}$ \\
eq27-12w-basic & 9 & 121 & 18 & 726 & $11 \mathrm{~s}$ \\
\hline
\end{tabular}


We now give some information about the examples we have used for the experiments. See the Appendix of the paper for an explicit description of the test set. All considered examples are non-linear systems of ordinary or partial difference equations with constant coefficients which are of interest in literature. For instance, the tests falkow are obtained by the discretization of the Falkowich-Karman equation which is a non-linear two-dimensional differential equation describing transonic flow in gas dynamics. The discretization we used are equations (41) in [11. Then the navier examples are based on equations $e_{1}, e_{2}, e_{3}, e_{4}$ of the system (13) in the paper [10] that are a finite difference scheme corresponding to the discretization (9) of the Navier-Stokes equations for two-dimensional viscous incompressible fluid flows. The tests heat are the discretization of the one-dimensional heat equation as described in the equations (10) and (11) of 22 . Finally, eq26 and eq27 are the equations (2.6) and (2.7) at page 24 of [14] which are examples of ordinary difference equations that have periodic solutions.

By analyzing the experiments, it is sufficiently clear that the strategy implemented in SigmaGBAsis is the safest one and hence on the average, the most efficient one. In fact, by decreasing the number of S-polynomials this strategy avoids the dramatical effects of involved reductions as for the tests falkow- $6 w$ and navier- $8 w$. For simpler examples the four strategies appear essentially equivalent. The algorithms SigmaGBASIS and SigmaGBAsis2 lead to practically identical computations but the latter method suffers from some overhead which is probably due to our still experimental implementation. For instance, even if the normal form modulo the ideal $N$ is described in the Definition 7.8, in our implementation we obtain it by computations, that is, by adding a Gröbner basis of $N$ to the input basis for SigmaGBASIS2.

The proposed algorithms usually provide only partial information about the structure of Gröbner $\Sigma$-bases since they are in general infinite. Nevertheless, it is interesting to note that by means of the finite $\Sigma$-criterion we have been able to certify that the examples falkow, navier and heat have finite bases with respect to the weight ranking. In particular, the elements of the Gröbner basis of falkow have maximum top order equal to 4 and hence they are certified in order 8 in about 4 minutes. The example navier has max top order equal to 6 and its certification is obtained in order 12 in less than one hour. Finally, the example heat has max top order 2 and it gets certification in order 4 in 0 seconds.

\section{Conclusions AND Future DiRECTiOns}

This paper shows that one cannot only generalize in a systematic way the Gröbner bases theory and related algorithms to the algebras of partial difference polynomials but it also makes these methods really work by introducing suitable gradings for such algebras. In fact, weight and order functions provide a Noetherian subalgebra filtration that implies termination and completeness certification for actual computations that are performed within some bounded degree that is over a finite number of variables. We have then developed the first implementation of a variant of Buchberger's algorithm for systems of linear or non-linear partial difference equations. Even if this implementation is just experimental, the approach corresponding to the algorithm SigmaGBASIS is strong enough to let it be able to work with discretizations of real world systems of non-linear differential equations. 
In this paper we consider difference equations with constant coefficients and hence a next step along this line of research is to extend the proposed methods to systems of difference equations with non-constant coefficients, that is, to assume that $\Sigma$ acts on the base field $K$ in a non-trivial way. Moreover, since the algebras of partial difference polynomials are free objects in the category of commutative algebras endowed with the action of a monoid $\Sigma$ isomorphic to $\mathbb{N}^{r}$, a natural future direction consists in extending the ideas introduced here to other types of monoid symmetry over commutative algebras as the ones used, for instance, in algebraic statistic [4, 16]. Starting from Gröbner bases, classical directions are the computation of the Hilbert series and free resolutions that one may generalize to partial difference ideals or other types of invariant ideals. Finally, we aim to have the proposed algorithms implemented in the kernel of computer algebra systems in order to tackle involved problems related with the discretization of systems of partial differential equations $9,11,12$.

\section{APPENDIX: THE TEST SET}

\section{Falkovich-Karman (falkow)}

The base field is $F=\mathbb{Q}(h, \tau, K, \gamma)$ (field of rational functions with rational coefficients) where $h$ is the space mesh step, $\tau$ is the time mesh step and $K, \gamma$ are parameters of the corresponding differential equation. The algebra of partial difference polynomials is $P=F\left[\varphi_{x}(i, j, k), \varphi_{y}(i, j, k), \varphi_{t}(i, j, k), \varphi(i, j, k) \mid i, j, k \geq 0\right]$. The monomial ordering of $P$ is the lexicographic one based on the following weight ranking:

$$
\begin{aligned}
& \ldots \succ \varphi_{x}(2,0,0) \succ \varphi_{y}(2,0,0) \succ \varphi_{t}(2,0,0) \succ \varphi(2,0,0) \succ \varphi_{x}(1,1,0) \\
& \succ \varphi_{y}(1,1,0) \succ \varphi_{t}(1,1,0) \succ \varphi(1,1,0) \succ \varphi_{x}(0,2,0) \succ \varphi_{y}(0,2,0) \succ \varphi_{t}(0,2,0) \\
& \succ \varphi(0,2,0) \succ \varphi_{x}(1,0,1) \succ \varphi_{y}(1,0,1) \succ \varphi_{t}(1,0,1) \succ \varphi(1,0,1) \succ \varphi_{x}(0,1,1) \\
& \succ \varphi_{y}(0,1,1) \succ \varphi_{t}(0,1,1) \succ \varphi(0,1,1) \succ \varphi_{x}(0,0,2) \succ \varphi_{y}(0,0,2) \succ \varphi_{t}(0,0,2) \\
& \succ \varphi(0,0,2) \succ \varphi_{x}(1,0,0) \succ \varphi_{y}(1,0,0) \succ \varphi_{t}(1,0,0) \succ \varphi(1,0,0) \succ \varphi_{x}(0,1,0) \\
& \succ \varphi_{y}(0,1,0) \succ \varphi_{t}(0,1,0) \succ \varphi(0,1,0) \succ \varphi_{x}(0,0,1) \succ \varphi_{y}(0,0,1) \succ \varphi_{t}(0,0,1) \\
& \succ \varphi(0,0,1) \succ \varphi_{x}(0,0,0) \succ \varphi_{y}(0,0,0) \succ \varphi_{t}(0,0,0) \succ \varphi(0,0,0),
\end{aligned}
$$

or on the following index ranking

$$
\begin{aligned}
& \ldots \succ \varphi_{x}(2,0,0) \succ \varphi_{x}(1,1,0) \succ \varphi_{x}(0,2,0) \succ \varphi_{x}(1,0,1) \succ \varphi_{x}(0,1,1) \\
& \succ \varphi_{x}(0,0,2) \succ \varphi_{x}(1,0,0) \succ \varphi_{x}(0,1,0) \succ \varphi_{x}(0,0,1) \succ \varphi_{x}(0,0,0) \\
& \succ \ldots \succ \varphi_{y}(2,0,0) \succ \varphi_{y}(1,1,0) \succ \varphi_{y}(0,2,0) \succ \varphi_{y}(1,0,1) \succ \varphi_{y}(0,1,1) \\
& \succ \varphi_{y}(0,0,2) \succ \varphi_{y}(1,0,0) \succ \varphi_{y}(0,1,0) \succ \varphi_{y}(0,0,1) \succ \varphi_{y}(0,0,0) \\
& \succ \ldots \succ \varphi_{t}(2,0,0) \succ \varphi_{t}(1,1,0) \succ \varphi_{t}(0,2,0) \succ \varphi_{t}(1,0,1) \succ \varphi_{t}(0,1,1) \\
& \succ \varphi_{t}(0,0,2) \succ \varphi_{t}(1,0,0) \succ \varphi_{t}(0,1,0) \succ \varphi_{t}(0,0,1) \succ \varphi_{t}(0,0,0) \\
& \succ \ldots \succ \varphi(2,0,0) \succ \varphi(1,1,0) \succ \varphi(0,2,0) \succ \varphi(1,0,1) \succ \varphi(0,1,1) \\
& \succ \varphi(0,0,2) \succ \varphi(1,0,0) \succ \varphi(0,1,0) \succ \varphi(0,0,1) \succ \varphi(0,0,0) .
\end{aligned}
$$


The input partial difference polynomials for the computational experiments are:

$$
\begin{aligned}
E_{1}= & -4 h p(2,1,1)-h \tau(\gamma+1) p_{x}(2,1,0)^{2}+2 h \tau K p_{x}(2,1,0)+4 h p(2,1,0) \\
& +2 h \tau p_{y}(1,2,0)-4 h^{2} p_{t}(1,1,0)+4 h p(0,1,1)-2 h \tau p_{y}(1,0,0) \\
& +h \tau(\gamma+1) p_{x}(0,1,0)^{2}-2 h \tau K p_{x}(0,1,0)-4 h p(0,1,0), \\
E_{2}= & \frac{h}{2}\left(p_{x}(1,0,0)+p_{x}(0,0,0)\right)-p(1,0,0)+p(0,0,0), \\
E_{3}= & \frac{h}{2}\left(p_{y}(0,1,0)+p_{y}(0,0,0)\right)-p(0,1,0)+p(0,0,0), \\
E_{4}= & 2 \tau p_{t}(0,0,1)-p(0,0,2)+p(0,0,0) .
\end{aligned}
$$

\section{Navier-Stokes (navier)}

The base field is $K=\mathbb{Q}(h, \tau, R e)$ where $R e$ is the Reynolds number. The algebra of partial difference polynomials is $P=K[p(i, j, k), u(i, j, k), v(i, j, k) \mid i, j, k \geq 0]$. The lexicographic monomial ordering of $P$ is defined by following weight ranking:

$$
\begin{aligned}
& \ldots \succ p(2,0,0) \succ u(2,0,0) \succ v(2,0,0) \succ p(1,1,0) \succ u(1,1,0) \succ v(1,1,0) \\
& \succ p(0,2,0) \succ u(0,2,0) \succ v(0,2,0) \succ p(1,0,1) \succ u(1,0,1) \succ v(1,0,1) \succ p(0,1,1) \\
& \succ u(0,1,1) \succ v(0,1,1) \succ p(0,0,2) \succ u(0,0,2) \succ v(0,0,2) \succ p(1,0,0) \succ u(1,0,0) \\
& \succ v(1,0,0) \succ p(0,1,0) \succ u(0,1,0) \succ v(0,1,0) \succ p(0,0,1) \succ u(0,0,1) \succ v(0,0,1) \\
& \succ p(0,0,0) \succ u(0,0,0) \succ v(0,0,0),
\end{aligned}
$$

or by the following index ranking:

$$
\begin{aligned}
& \ldots \succ p(2,0,0) \succ p(1,1,0) \succ p(0,2,0) \succ p(1,0,1) \succ p(0,1,1) \succ p(0,0,2) \\
& \succ p(1,0,0) \succ p(0,1,0) \succ p(0,0,1) \succ p(0,0,0) \succ \ldots \succ u(2,0,0) \succ u(1,1,0) \\
& \succ u(0,2,0) \succ u(1,0,1) \succ u(0,1,1) \succ u(0,0,2) \succ u(1,0,0) \succ u(0,1,0) \\
& \succ u(0,0,1) \succ u(0,0,0) \succ \ldots \succ v(2,0,0) \succ v(1,1,0) \succ v(0,2,0) \succ v(1,0,1) \\
& \succ v(0,1,1) \succ v(0,0,2) \succ v(1,0,0) \succ v(0,1,0) \succ v(0,0,1) \succ v(0,0,0) .
\end{aligned}
$$

The input partial difference polynomials are

$$
\begin{aligned}
E_{1}= & 2 h(u(0,2,1)-u(0,0,1)+v(0,1,2)-v(0,1,0)), \\
E_{2}= & -4 \frac{\tau}{R e} h^{2}(u(0,4,2)+u(0,2,4))+16 h^{4} u(1,2,2)+8 \tau h^{3}\left(u(0,3,2)^{2}\right. \\
& +u(0,2,3) v(0,2,3)+p(0,3,2))+16 h^{2}\left(-h^{2}+\frac{\tau}{R e}\right) u(0,2,2)-8 \tau h^{3}\left(u(0,1,2)^{2}\right. \\
& +u(0,2,1) v(0,2,1)+p(0,1,2))-4 \frac{\tau}{R e} h^{2}(u(0,0,2)+u(0,2,0)), \\
E_{3}= & -4 \frac{\tau}{R e} h^{2}(v(0,4,2)+v(0,2,4))+16 h^{4} v(1,2,2)+8 \tau h^{3}\left(v(0,2,3)^{2}\right. \\
& +u(0,3,2) v(0,3,2)+p(0,2,3))+16 h^{2}\left(-h^{2}+\frac{\tau}{R e}\right) v(0,2,2)-8 \tau h^{3}\left(v(0,2,1)^{2}\right. \\
& +u(0,1,2) v(0,1,2)+p(0,2,1))-4 \frac{\tau}{R e} h^{2}(v(0,2,0)+v(0,0,2)), \\
E_{4}= & 4 h^{2}\left(v(0,2,4)^{2}+u(0,4,2)^{2}+v(0,2,0)^{2}+u(0,0,2)^{2}+p(0,4,2)\right. \\
& +p(0,2,4)+p(0,2,0)+p(0,0,2))+8 h^{2}\left(u(0,3,3) v(0,3,3)-v(0,2,2)^{2}\right. \\
& \left.-u(0,2,2)^{2}-u(0,3,1) v(0,3,1)-u(0,1,3) v(0,1,3)+u(0,1,1) v(0,1,1)\right) \\
& -16 h^{2} p(0,2,2) .
\end{aligned}
$$

Heat (heat)

The base field is by definition $K=\mathbb{Q}(h, \tau)$ and the partial difference polynomial algebra is $P=K[x(i, j), t(i, j), u(i, j) \mid i, j \geq 0]$. The lexicographic monomial order of $P$ is obtained by the following weight ranking:

$$
\begin{aligned}
& \ldots \succ x(2,0) \succ t(2,0) \succ u(2,0) \succ x(1,1) \succ t(1,1) \succ u(1,1) \succ x(0,2) \\
& \succ t(0,2) \succ u(0,2) \succ x(1,0) \succ t(1,0) \succ u(1,0) \succ x(0,1) \succ t(0,1) \succ u(0,1) \\
& \succ x(0,0) \succ t(0,0) \succ u(0,0) .
\end{aligned}
$$


The input is given by

$$
\begin{aligned}
E_{1}= & (u(1,0)-u(0,0))(x(0,1)-x(0,0))^{2}-(u(0,2)-2 u(0,1) \\
& +u(0,0))(t(1,0)-t(0,0)) \\
E_{2}= & x(1,0)-x(0,0), E_{3}=x(0,1)-x(0,0)-h, \\
E_{4}= & t(1,0)-t(0,0)-\tau, E_{5}=t(0,1)-t(0,0) .
\end{aligned}
$$

\section{Equation $2.6(e q 26)$}

One considers the algebra of ordinary difference polynomials $P=\mathbb{Q}[x(i) \mid i \geq 0]$ endowed with the lexicographic monomial ordering such that

$$
\ldots \succ x(4) \succ x(3) \succ x(2) \succ x(1) \succ x(0) .
$$

The input ordinary difference polynomial is

$$
E=x(3) x(0)-x(2)-x(1)-1 .
$$

\section{Equation 2.7 (eq27)}

We consider the same algebra $P=\mathbb{Q}[x(i) \mid i \geq 0]$ endowed with the same monomial ordering as in the previous example. The input polynomial here is

$$
E=x(4) x(2) x(0)-x(3) x(1) .
$$

\section{ACKNOWLEDGMENTS}

The author would like to thank Vladimir Gerdt for introducing him to the theory of difference algebras and supporting the preparation of testing examples. He is also grateful to the research group of Singular [7] for the courtesy of allowing access to their servers for performing computational experiments. Thanks also to the reviewer for all valuable remarks that have helped to make the paper more readable.

\section{REFERENCES}

[1] Matthias Aschenbrenner and Christopher J. Hillar, Finite generation of symmetric ideals, Trans. Amer. Math. Soc. 359 (2007), no. 11, 5171-5192, DOI 10.1090/S0002-9947-07-041165. MR2327026 (2008g:13030)

[2] George M. Bergman, The diamond lemma for ring theory, Adv. in Math. 29 (1978), no. 2, 178-218, DOI 10.1016/0001-8708(78)90010-5. MR506890(81b:16001)

[3] A. M. Bigatti, M. Caboara, and L. Robbiano, Computing inhomogeneous Gröbner bases, J. Symbolic Comput. 46 (2011), no. 5, 498-510, DOI 10.1016/j.jsc.2010.10.002. MR 2781934 (2012c:13061)

[4] Andries E. Brouwer and Jan Draisma, Equivariant Gröbner bases and the Gaussian twofactor model, Math. Comp. 80 (2011), no. 274, 1123-1133, DOI 10.1090/S0025-5718-201002415-9. MR2772115 (2012g:13049)

[5] B. Buchberger, Ein algorithmisches Kriterium für die Lösbarkeit eines algebraischen Gleichungssystems (German), Aequationes Math. 4 (1970), 374-383. MR0268178 (42 \#3077)

[6] Richard M. Cohn, Difference Algebra, Interscience Publishers John Wiley \& Sons, New YorkLondon-Sydeny, 1965. MR0205987 (34 \#5812)

[7] W. Decker, G.-M. Greuel, G. Pfister, and H. Schönemann, Singular 3-1-5 - A computer algebra system for polynomial computations (2012). http://www.singular.uni-kl.de

[8] David Eisenbud, Commutative Algebra, Graduate Texts in Mathematics, vol. 150, SpringerVerlag, New York, 1995. With a view toward algebraic geometry. MR1322960 (97a:13001)

[9] V. P. Gerdt, Consistency analysis of finite difference approximations to PDE systems. In: Adam G. et al. (Eds.), Proc. of Mathematical Modeling and Computational Physics. MMCP 2011, 28-42, Lecture Notes in Comput. Sci., 7175, Springer, Heidelberg, 2012. 
[10] V. P. Gerdt and Y. A. Blinkov, Involution and difference schemes for the Navier-Stokes equations. In: Gerdt V.P. et al. (Eds.), Proc. of Computer Algebra in Scientific Computing. CASC 2009, 94-105, Lecture Notes in Comput. Sci., 5743, Springer, Berlin, 2009.

[11] Vladimir P. Gerdt, Yuri A. Blinkov, and Vladimir V. Mozzhilkin, Gröbner bases and generation of difference schemes for partial differential equations, SIGMA Symmetry Integrability Geom. Methods Appl. 2 (2006), Paper 051, 26, DOI 10.3842/SIGMA.2006.051. MR2240724 (2007h:65084)

[12] V. P. Gerdt and D. Robertz, A Maple package for computing Gröbner bases for linear recurrence relations. Nucl. Instrum. Methods, 599 (2006), 215-219. http://wwwb.math.rwthaachen.de/Janet

[13] Michel Gondran and Michel Minoux, Graphs, Dioids and Semirings, New Models and Algorithms. Operations Research/Computer Science Interfaces Series, vol. 41, Springer, New York, 2008. MR2389137(2009g:16066)

[14] E. A. Grove and G. Ladas, Periodicities in Nonlinear Difference Equations, Advances in Discrete Mathematics and Applications, vol. 4, Chapman \& Hall/CRC, Boca Raton, FL, 2005. MR2193366 (2006j:39002)

[15] Graham Higman, Ordering by divisibility in abstract algebras, Proc. London Math. Soc. (3) 2 (1952), 326-336. MR0049867 (14,238e)

[16] Christopher J. Hillar and Abraham Martín del Campo, Finiteness theorems and algorithms for permutation invariant chains of Laurent lattice ideals, J. Symbolic Comput. 50 (2013), 314-334, DOI 10.1016/j.jsc.2012.06.006. MR2996883

[17] E. R. Kolchin, Differential Algebra and Algebraic Groups, Academic Press, New York, 1973. Pure and Applied Mathematics, Vol. 54. MR0568864 (58 \#27929)

[18] R. La Scala, Extended letterplace correspondence for nongraded noncommutative ideals and related algorithms, preprint (2012), 1-20. arXiv:1206.6027

[19] Roberto La Scala and Viktor Levandovskyy, Letterplace ideals and non-commutative Gröbner bases, J. Symbolic Comput. 44 (2009), no. 10, 1374-1393, DOI 10.1016/j.jsc.2009.03.002. MR2543425 (2010m:16031)

[20] Roberto La Scala and Viktor Levandovskyy, Skew polynomial rings, Gröbner bases and the letterplace embedding of the free associative algebra, J. Symbolic Comput. 48 (2013), 110-131, DOI 10.1016/j.jsc.2012.05.003. MR2980469

[21] V. Levandovskyy and B. Martin, A symbolic approach to generation and analysis of finite difference schemes of partial differential equations. In: Langer U. et al. (Eds.), Numerical and Symbolic Scientific Computing: Progress and Prospects. Springer (2012), 123-156.

[22] Decio Levi, Lie symmetries for lattice equations, Note Mat. 23 (2004/05), no. 2, 139-156. MR2141114 (2006b:37157)

[23] Alexander Levin, Difference Algebra, Algebra and Applications, vol. 8, Springer, New York, 2008. MR2428839(2009m:12010)

[24] David A. Meyer and Noland Wallach, Invariants for multiple qubits: the case of 3 qubits, Mathematics of quantum computation, Comput. Math. Ser., Chapman \& Hall/CRC, Boca Raton, FL, 2002, pp. 77-97. MR2007943 (2004h:81034)

[25] J. F. Ritt, Differential Equations From the Algebraic Standpoint. Amer. Math. Soc. Colloquium Publ., Vol. 14, AMS, New York, 1932.

[26] Joseph Fels Ritt, Differential Algebra, American Mathematical Society Colloquium Publications, Vol. XXXIII, American Mathematical Society, New York, N. Y., 1950. MR0035763 $(12,7 \mathrm{c})$

[27] Werner M. Seiler, Involution, The formal theory of differential equations and its applications in computer algebra. Algorithms and Computation in Mathematics, vol. 24, Springer-Verlag, Berlin, 2010. MR2573958(2011h:35003)

Dipartimento di Matematica, via Orabona 4, 70125 Bari, Italia

E-mail address: roberto.lascala@uniba.it 